\title{
Bifurcation Analysis of Continuous Biochemical Reactor Models
}

\author{
Yongchun Zhang and Michael A. Henson*
}

Department of Chemical Engineering, Louisiana State University, Baton Rouge, Louisiana 70803-7303

\begin{abstract}
The validity of a biochemical reactor model often is evaluated by comparing transient responses to experimental data. Dynamic simulation can be a rather inefficient and ineffectivetool for analyzing bi oreactor models that exhibit complex nonlinear behavior. Bifurcation analysis is a powerful tool for obtaining a more efficient and complete characterization of the model behavior. To illustrate the power of bifurcation analysis, the steady-state and transient behavior of three continuous bioreactor models consisting of a small number of ordinary differential equations are investigated. Several important features, as well as potential limitations, that are difficult to ascertain via dynamic simulation are disclosed through the bifurcation analysis. The results motivate the use of dynamic simulation and bifurcation analysis as complementary tools for analyzing the nonlinear behavior of bioreactor models.
\end{abstract}

\section{Introduction}

Biochemical reactors can be viewed as highly complex dynamic systems. The intracellular and extracellular environments comprise many chemical components, and each cell has unique properties. A rigorous mechanistic model accounting for both these complexities is known as a structured and segregated model $(9,13,33)$. Such models consist of coupled sets of partial differential equations and ordinary differential equations. In addition to being difficult to formulate, these models are not amenable to systematic analysis as a result of their complexity. Simplified models can be devel oped either by neglecting the intracellular chemical structure (unstructured segregated models) $(6,12,17)$ or by neglecting heterogeneity of the cell population (structured unsegregated models) $(8,20,32)$. The simplest models are both unstructured and unsegregated (10). I f the intracellular environment can be characterized by a few critical variables $(20,32)$, then structured unsegregated models (like unstructured unsegregated models) consist of a reasonably small number of nonlinear ordinary differential equations. Such models are well suited for rigorous analysis.

Three unsegregated models of different cell cultures are studied in this paper. First the structured model of Hybridoma cells proposed by Guardia and co-workers (16) is considered. This mammalian cell culture is reported to exhibit multiple steady states under certain operating conditions $(14,38)$. The cybernetic modeling paradigm used to reproduce this behavior involves the maximization of cell growth via competition between alternative pathways that utilize a pair of complementary and partially substitutable substrates. An unstructured model proposed by McLellan and co-workers (27) for continuous fermentation of the microorganism Zymomonas mobiliz is studied next. Continuous cultures of this microorganism exhibit oscillatory behavior which adversely affects ethanol productivity and reactor operability. This behavior is captured in the model by introducing a dynamic

* Ph: 225-388-3690. Fax: 225-388-1476. E-mail: henson@ che.lsu.edu. specific growth rate that accounts for the inhibitory effect of the past ethanol rate of change on the current state of the reactor. The last model investigated is a structured model proposed by J ones and Kompala (20) for the yeast Saccharomyces cerevisiae. Continuous cultures of this microorganism have been shown to exhibit complex dynamic behavior that includes the sudden appearance and disappearance of sustained oscillations $(29,34,35)$. The cybernetic model reproduces this behavior through the competition of three metabolic pathways that are utilized to maximize the cell growth rate.

Transient bioreactor models often are evaluated by comparing experimental data and model simulation results. While dynamic simulation is a very useful tool for model validation, several limitations can be identified: (i) it is inefficient and potentially inconclusive, especially when the model possesses slow dynamic modes; (ii) it is necessarily incomplete since only a limited number of simulation tests can be performed and important dynamic behaviors may not be observed; and (iii) it does not easily reveal the model characteristics that lead to certain dynamic behaviors. Therefore, dynamic simulation should not be viewed as the only tool for evaluating transient bioreactor models.

The purpose of this paper is to demonstrate that bifurcation analysis is a powerful tool for evaluating transient models of continuous bioreactor. The objective of bifurcation theory is to characterize changes in the qualitative dynamic behavior of a nonlinear system as key parameters are varied. The model equations are used to locate steady-state solutions, periodic solutions, and bifurcation points where the qualitative dynamic behavior changes. Bifurcation analysis can be much more effective than simply integrating the model equations over time and comparing the transient responses to experimental data. Instead a "complete" picture of the model behavior is obtained in the form of a bifurcation diagram. This diagram can be used to determine if the model supports the steady-state and dynamic behavior observed experimentally. It also can guide the design of experiments aimed at validating unexpected model predictions. Numerical bifurcation packages developed by a 
number of researchers $(11,21,23)$ make bifurcation analysis of low-order nonlinear systems reasonably simple.

It is important to note that a number of other investigators have applied bifurcation analysis to continuous bioreactor models $(1,26,30,37)$. Many of these studies focus on the dynamics of two microbial populations competing for a common rate-limiting substrate $(2,3,24$, 31). In most studies the objective is to provide a very detailed characterization of the model behavior with mi nimal comparison to available experimental data. The objective of this paper is notably different. We are concerned primarily with the use of bifurcation analysis for validation of transient bioreactor models. We emphasize the comparison of physically meaningful model behavior with experimental data rather than cataloging all possible model behaviors. A similar approach has been pursued by J ones and Kompala (20) to determine unknown parameters in their cybernetic yeast model. The present contribution can be viewed as an extension of our bifurcation studies on unstructured cell population balance models for continuous yeast bioreactors (37).

The remainder of the paper is organized as follows. Basic concepts of bifurcation theory and an introduction to the bifurcation package AUTO are presented in Section 2. The dynamic behavior and bifurcation analysis of the aforementioned bioreactor models are discussed in Sections 3-5. A summary and conclusions are presented in Section 6.

\section{Bifurcation Analysis}

A nonlinear dynamic system differs from a linear dynamic system in that its qualitative properties can change under small perturbations of the model parameters. These properties include the number of equilibria, stability of the equilibria, existence of limit cycles, multiple modes of behavior, and chaos (22). Below we provide a very brief introduction to bifurcation analysis. The textbook by K uznetsov (22) provides more complete descriptions of these concepts. A bifurcation is introduced formally as follows.

Definition 1. Two dynamical system $F: R \quad n \rightarrow R$ t and $G: R{ }^{n} \rightarrow R$ t are called topologically equivalent if there exists a diffeomorphism $h: R{ }^{n} \rightarrow R \quad n$ such that $h \cdot F=$ $\mathrm{G} \cdot \mathrm{h}$.

Two topologically equivalent systems have the same qual itative dynamic behavior in the sense that they can be mapped to each other.

Definition 2. The appearance of a topologically nonequivalent phase portrait under variation of a parameter is called a bifurcation.

Bifurcation analysis is the study of how the qualitative properties of a nonlinear dynamic system change as key parameters are varied. Consider a continuous-time nonlinear system depending on a parameter vector $\alpha$ :

$$
x=f(x, \alpha), \quad x \in R \quad n, \alpha \in R \quad
$$

where $f$ is smooth with respect to both the state vector $x$ and the bifurcation parameter vector $\alpha$. If $x_{0}$ is a hyperbolic equilibrium point where all the real parts of the eigenvalues of the Jacobian matrix Df $\left(\mathrm{x}_{0}\right)$ are nonzero, then a small perturbation in the model parameters will not change the qualitative behavior of the system, i.e., a hyperbolic equilibrium is structurally stable. Bifurcations occur when some of the eigenvalues approach the imaginary axis in the complex plane. The simplest bifurcations are associated with a single real eigenvalue becoming zero $\left(\lambda_{1}=0\right)$ or a pair of complex conjugate eigenvalues crossing the imaginary axis $\left(\lambda_{1,2}\right.$ $\left.= \pm \mathrm{i} \omega_{0}, \omega_{0}>0\right)$.

Definition 3. The bifurcation where $\lambda_{1}=0$ is called a fold bifurcation.

Definition 4. The bifurcation where $\lambda_{1,2}= \pm \mathrm{i} \omega_{0}, \omega_{0}>$ 0 is called a Hopf bifurcation.

These are the most common bifurcations present in nonlinear systems. Fold bifurcations usually are the cause of multiple steady states and hysteresis behavior. Hopf bifurcations are responsible for the appearance and disappearance of periodic solutions.

While bifurcation theory is a powerful tool for model characterization, analytical treatment of physically based models usually is intractable because of the complexity of the nonlinear model equations. A number of software packages including AUTO (11), LOCBIF (21), and CONTENT (23) have been developed for numerical bifurcation analysis of low-order nonlinear models. Numerical bifurcation analysis involves an iterative procedure known as continuation. First a stable steady-state or periodic solution for a particular set of parameter values is located by dynamic simulation. Then one of the parameters is varied to allow the continued calculation of solutions as a function of this bifurcation parameter. At each iteration, a step in the bifurcation parameter is taken and a predictor-corrector method is utilized to locate the new solution. The step size of each iteration is controlled by a convergence criteria. The procedure is repeated until a desired range of parameter values has been evaluated. Therefore, continuation can provide a "complete" picture of the nonlinear dynamic behavior.

The results of the continuation calculations typically are presented as a bifurcation diagram where the behavior of a key model variable is shown as a function of the bifurcation parameter. The steady-state and periodic solutions are mapped into this two-dimensional space. As compared to dynamic simulation, a key advantage of continuation is that unstable as well as stable solutions can be located. Because it provides a very concise and complete description of the system behavior, a bifurcation diagram is ideal for comparing model predictions to experimentally observed behavior. For example, the range of model parameter values that support multiple steady states or periodic solutions can be determined. This allows a more meaningful analysis of a nonlinear model than is possible with dynamic simulation alone. In the next three sections, the applicability of bifurcation analysis to continuous bioreactor models is demonstrated via three example systems.

The AUTO continuation package developed by Doedel and co-workers (11) is perhaps the most widely used numerical bifurcation code. AUTO can perform bifurcation analysis of nonlinear systems described by algebraic equations, ordinary differential equations, and parabolic partial differential equations. In addition to the simple fold and Hopf bifurcations described above, AUTO can locate tori and period doubling bifurcations as a function of two or more parameters. AUTO also includes a graphical user interface (GUI) which simplifies specification of computational parameters required by the continuation code. For these reasons, we use AUTO in this paper. It is important to note that AUTO and other general purpose bifurcation codes can be expected to work only for nonlinear models of moderate dimension. There fore, they are best suited for analysis of unstructured, unsegregated and simple structured, unsegregated biore actor models. 


\section{Hybridoma Cell Bioreactor Model}

Background. Hybridoma cells utilize glucose and glutamine as complementary and partially substitutable substrates for growth $(4,36)$. There exists several metabolic pathways, each of which is favored under certain culture conditions, for cell growth. The existence of these multiple pathways creates complex behavior when $\mathrm{Hy}$ bridoma cells are grown in a continuous bioreactor. In particular, researchers have shown that different steady states can be reached when cultures with the same operating conditions but different initial metabolic states are switched from batch or fed-batch mode to continuous operation $(14,38)$.

A structured, unsegregated model has been proposed by Guardia et al. (16) to capture the observed steadystate multiplicity. The cybernetic model accounts for the multiple metabolic pathways created by the complementary and partially substitutable substrate utilization. The cybernetic model predicts the existence of multiple steady-state solutions for some operating conditions. More specifically, it is shown that different initial conditions established by batch and fed-batch operation can lead to different steady-state solutions. However, the authors note that this property is very sensitive to changes in the model parameters and the region of operating conditions that support multiplicity is quite small. It is not clear if this lack of robustness is attributable to the model structure or to the particular choice of model parameters. Furthermore, a precise characterization of the operating range which supports steady-state multiplicity has not been presented.

Transient Model. Ramkrishna and co-workers (32) have proposed that microorganisms optimize utilization of available substrates to maximize their instantaneous growth rate. While somewhat controversial, this hypothesis has been derived from the analysis of extensive experimental data. The cybernetic modeling approach has been used to capture the partially substitutable and complementary substrate utilization that leads to multiple metabolic pathways in Hybridoma cultures. A detailed description of the metabolic pathways can be found in the original reference (16). The cybernetic model includes two substrates, glucose and glutamine; three intermediates; and five enzymes associated with the various pathways.

The cybernetic model equations are

$$
\begin{aligned}
\frac{d X}{d t} & =\left(r_{g}-D\right) X \\
\frac{d S_{1}}{d t} & =-\left(r_{1} v_{1}^{s} v_{1}^{c}+r_{3} v_{3}^{c}\right) X+D\left(S_{1}^{f}-S_{1}\right) \\
\frac{d S_{2}}{d t} & =-r_{2} v_{2}^{s} X+D\left(S_{2}^{f}-S_{2}\right) \\
\frac{d M_{1}}{d t} & =Y_{1} r_{1} v_{1}^{s} v_{1}^{c}+Y_{4} r_{5} v_{5}^{s}-\left(Y_{m_{1} x}+M_{1}\right) r_{g}-r_{4} v_{4}^{s} \\
\frac{d M_{2}}{d t} & =Y_{2} r_{2} v_{2}^{s}+Y_{5} r_{4} v_{4}^{s}-\left(Y_{m_{2} x}+M_{2}\right) r_{g}-r_{5} v_{5}^{s} \\
\frac{d M_{3}}{d t} & =Y_{3} r_{3} v_{3}^{c}-\left(Y_{m_{3} x}+M_{3}\right) r_{g} \\
\frac{d e}{d t} & =r_{e}^{*}+r_{e} u_{i}^{c} u_{i}^{s}-\left(r_{g}+b_{i}\right) e_{1} ; i=1, \ldots, 5
\end{aligned}
$$

where $X$ is the cell mass concentration; $S_{1}$ and $S_{2}$ are the
Table 1. Hybridoma Cell Reactor Model Parameters

\begin{tabular}{cll}
\hline parameter & \multicolumn{1}{c}{ unit } & \multicolumn{1}{c}{ value } \\
\hline$r_{i}^{\max }$ & $g / g d w-h$ & $0.05,0.03,0.01,0.03,0.01$ \\
$\mathrm{~K}_{\mathrm{i}}$ & $\mathrm{g} / \mathrm{L}$ & $0.001,0.001,0.001,0.01,0.0001$ \\
$\mathrm{r}_{\mathrm{e}}^{\max }$ & $\mathrm{g} / \mathrm{gdw}-\mathrm{h}$ & $0.001,0.0005,0.001,0.0005,0.001$ \\
$\mathrm{~K}_{\mathrm{e}}$ & $\mathrm{g} / \mathrm{L}$ & $0.001,0.0001,0.001,1 \mathrm{e}-5,1 \mathrm{e}-5$ \\
$\mathrm{r}_{\mathrm{g}}^{*}$ & $\mathrm{~g} / \mathrm{gdw}-\mathrm{h}$ & $1 \mathrm{e}-6,1 \mathrm{e}-6,1 \mathrm{e}-6,1 \mathrm{e}-6,1 \mathrm{e}-6$ \\
$\mathrm{~b}_{\mathrm{i}}^{*}$ & $\mathrm{gdw} / \mathrm{gdw}-\mathrm{h}$ & $0.05,0.1,0.1,0.1,0.05$ \\
$\mathrm{Y}_{\mathrm{i}}$ & $\mathrm{g} / \mathrm{g}$ & $0.9,0.9,0.8,1.0,1.0$ \\
$\mathrm{~K}_{g M_{i}}$ & $\mathrm{~g} / \mathrm{gdw}$ & $0.0005,0.0005,0.0005$ \\
$\mathrm{Y}_{\mathrm{M}_{\mathrm{i}}}$ & $\mathrm{g} / \mathrm{gdw}$ & $0.7,0.99,0.1$ \\
$\mathrm{r}_{\mathrm{g}}^{\max }$ & $\mathrm{gdw} / \mathrm{gdw}-\mathrm{h}$ & 0.0575 \\
& &
\end{tabular}

concentrations of glucose and glutamine, respectively; $\mathrm{M}_{\mathrm{i}}$ and $\mathrm{e}$ are the concentrations of the three intermediates and five enzymes, respectively; $S_{i}^{f}$ are the feed substrate concentrations; $D$ is the dilution rate; $Y_{i}$ are the yield coefficients; $r_{e}^{*}$ is the constitutive synthesis rate of the enzyme e; and $b_{i}$ is the degradation rate constant of the enzyme e. The cybernetic variables $u_{i}^{c}, u_{i}^{s}, v_{i}^{c}$ and $v_{i}^{s}$ are the synthesis and activity coefficients of the enzymes for the complementary and substitutable pathways, respectively. The reaction rates $r_{i}$ and $r_{e}$ are assumed to follow Monod-type kinetics. Their definitions can be found in the origi nal reference (16) and are omitted here for sake of brevity.

We experienced some difficulties producing multiple steady-state solutions with the model parameter values reported in ref 16. After modifying several parameter values, we were able to generate the expected multiple steady-state behavi or. The parameter values used in our simulation and bifurcation studies are listed in Table 1 where the values of $r_{i}^{\max }$ and $Y_{M_{i} x}$ are different from those listed in the original reference. The discrepancy between the two sets of parameter values further motivates the need for a more detailed investigation of the model behavior using bifurcation analysis.

Results and Discussion. Several dynamic simulation tests were performed to study the steady-state behavior of the cybernetic model in different regions of the operating space. In the first test, the reactor initially is at a steady state corresponding to $S_{1}^{f}=0.95 \mathrm{~g} / \mathrm{L}, \mathrm{S}_{2}^{f}=0.43$ $\mathrm{g} / \mathrm{L}$, and $\mathrm{D}=0.0295 \mathrm{~h}^{-1}$. The results obtained for step changes of various magnitudes in the dilution rate are shown in Figure 1 where the cell mass concentration (X) is selected as a representative output variable for the culture. The steady states obtained for $\mathrm{D}=0.0300 \mathrm{~h}^{-1}$ and $D=0.0292 \mathrm{~h}^{-1}$ are close to the initial steady state, while those for $D=0.0291 h^{-1}$ and $D=0.0290 h^{-1}$ are very far removed. Also shown is the response obtained for a step change to $D=0.0300 \mathrm{~h}^{-1}$ at $\mathrm{t}=2000 \mathrm{~h}$ from the $\mathrm{D}=0.0290 \mathrm{~h}^{-1}$ steady state. The culture reaches a different steady state than the one obtained for the $\mathrm{D}=$ $0.0300 \mathrm{~h}^{-1}$ step change from the initial steady state.

These dynamic simulation results demonstrate that the cybernetic model predicts the presence of multiple stable steady states and hysteresis behavior. On the other hand, this type of analysis provides a rather incomplete picture of the model behavior. For instance, dynamic simulation is not a convenient tool for determining the range of dilution rates over which multiple steady states exist. This is very valuable information for model validation. Below we show that bifurcation analysis is a powerful tool for extracting this knowledge.

Figure 2 shows the one-parameter bifurcation diagram for the cybernetic model where the dilution rate $(D)$ is chosen as the bifurcation parameter and the cell mass 


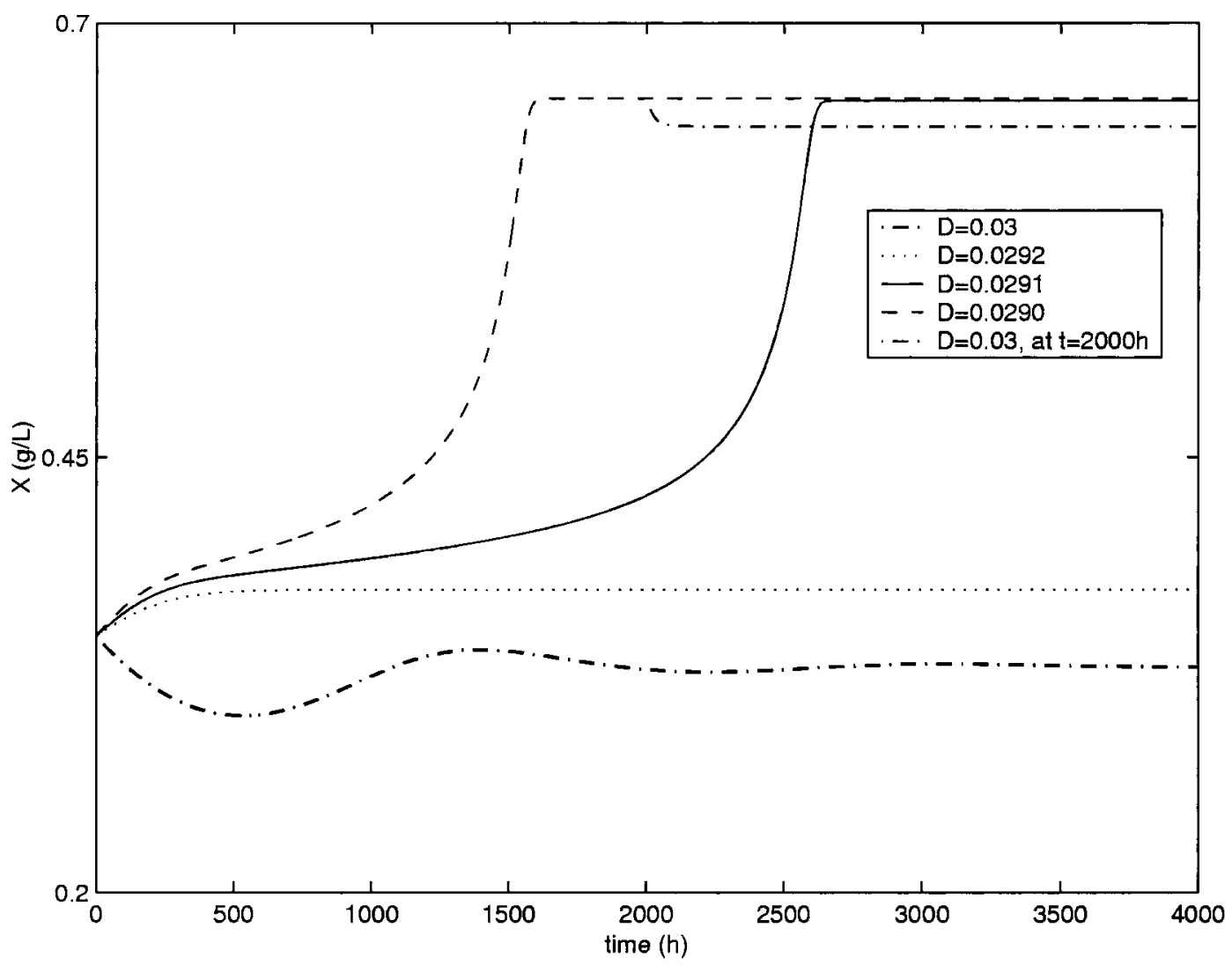

Figure 1. Dynamic simulation of the Hybridoma reactor model.

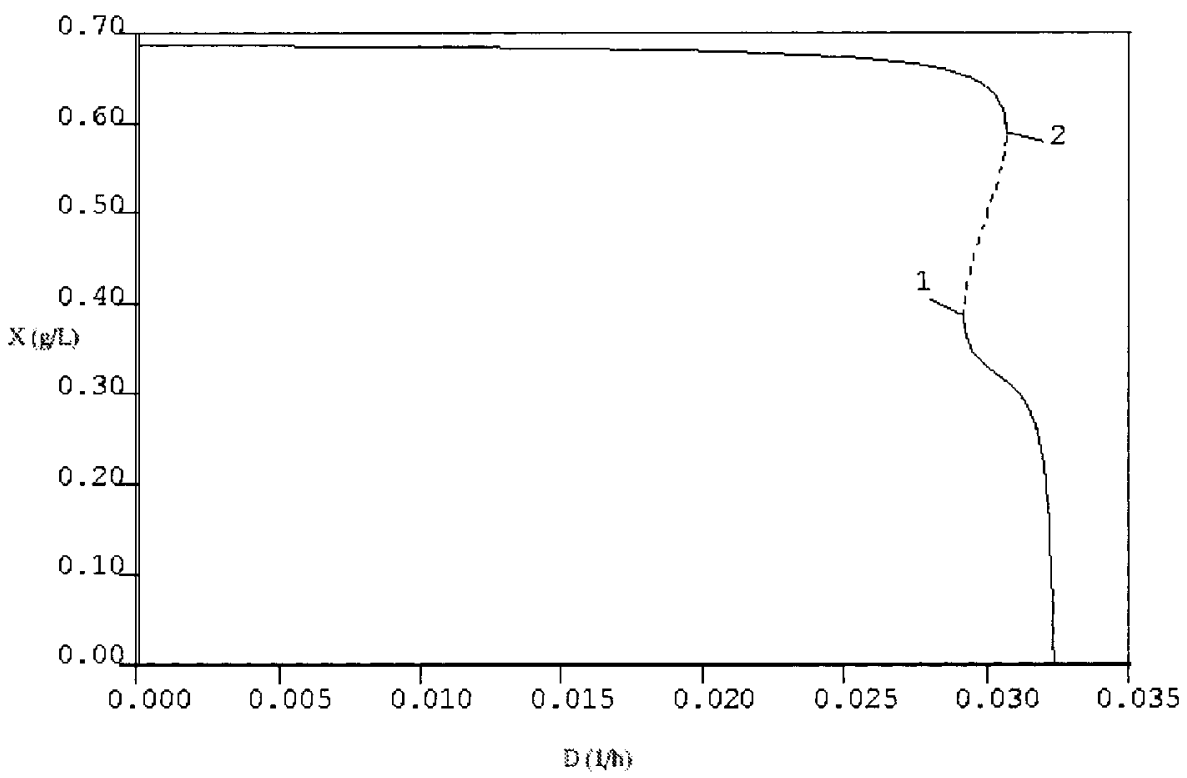

Figure 2. Bifurcation diagram of the Hybridoma reactor model.

concentration $(X)$ is the output variable. The feed substrate concentrations are held constant at $S_{1}^{f}=0.95 \mathrm{~g} / \mathrm{L}$ and $S_{2}^{f}=0.43 \mathrm{~g} / \mathrm{L}$. The locus of steady-state operating points as a function of $D$ is determined using AUTO. The solid line (-) denotes stable steady-state solutions, while the dashed line (-- ) denotes unstable steady-state solutions. The model exhibits fold bifurcations at $D=$ $0.02917 \mathrm{~h}^{-1}$ (point 1) and D $=0.03068 \mathrm{~h}^{-1}$ (point 2), which delineate the parameter space where multiple steadystate solutions exists. In this region the model shows hysteresis behavior due to the S-shaped steady-state locus. There only is a single stable steady-state solution outside this region.
The bifurcation diagram clearly shows the existence of multiple steady-state solutions and provides an explanation for the hysteresis behavior observed in Figure 1. Each stable steady state has a domain of attraction from which all initial conditions converge to that steady state. Initial conditions corresponding to low dilution rates converge to the upper steady state while those corresponding to high dilution rates converge to the lower steady state. The bifurcation diagram also shows that the parameter region that supports multiple steady-state solutions is quite small: $D \in\left[0.02917 \mathrm{~h}^{-1}, 0.03068 \mathrm{~h}^{-1}\right]$. This is much more valuable information for model validation than simply knowing the model exhibits 


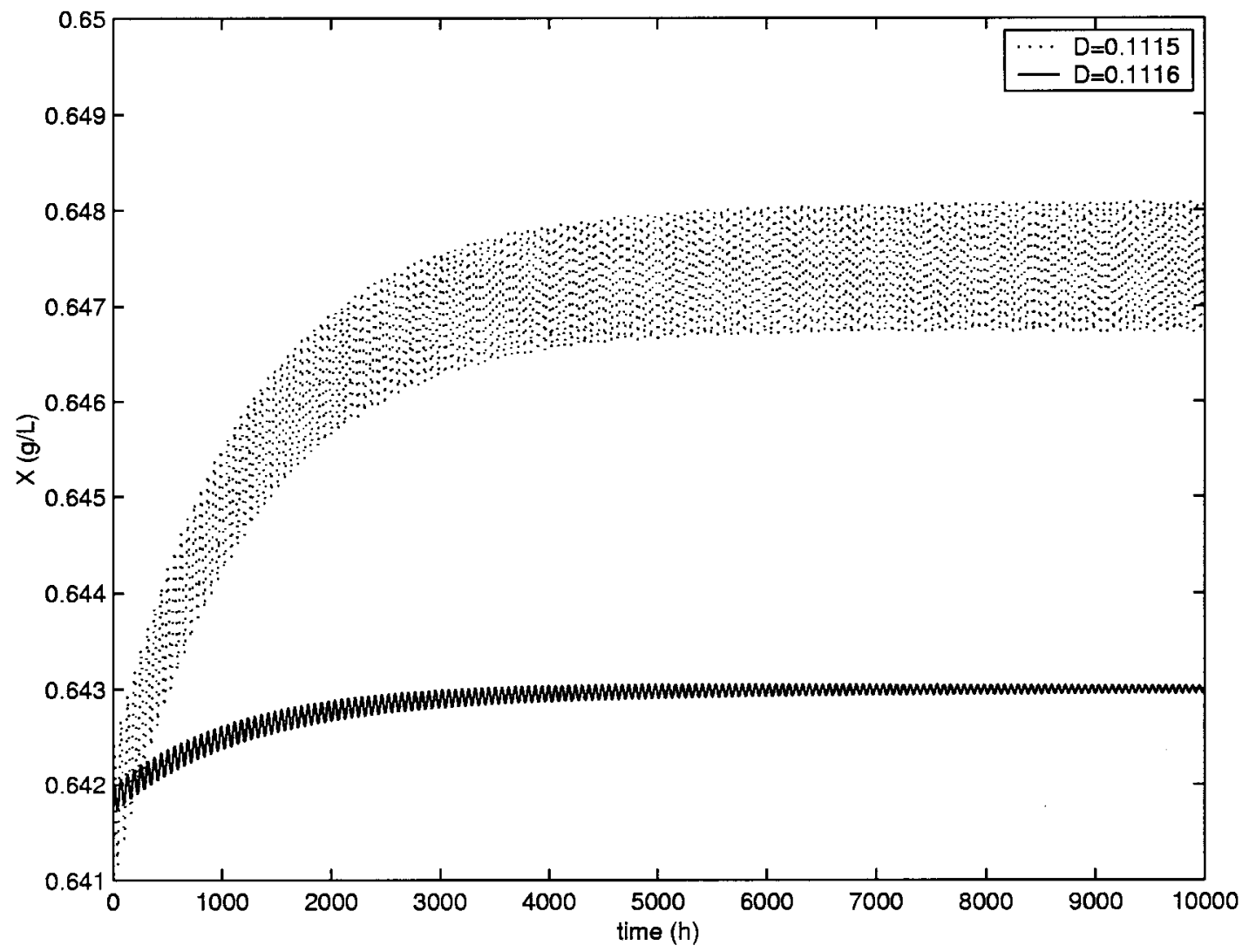

Figure 3. Dynamic simulation of the Zymomonas mobilis reactor model at high dilution rates.

multiple steady states. In particular, experimental determination of the dilution rates that support multiple steady states can be used to adjust the model parameters to obtain agreement. If this is not possible, the model structure may be concluded to be inadequate. Such conclusions are very difficult to obtain from dynamic simulation alone.

\section{Zymomonas mobilis Reactor Model}

Background. Zymomonas mobilis has been proposed as a more promising microorganism than conventional baker's yeast for industrial production of ethanol $(5,7)$. A major drawback of this microorganism is that it exhibits sustained oscillations over a wide range of operating conditions when grown in continuous culture. This leads to decreased ethanol productivity and less efficient use of available substrate $(7,15)$. Various models have been proposed to describe the oscillatory dynamics of continuous Zymomonas mobilis cultures (10, 15, 18, 25). Daugulis et al. (10) present an unstructured, unsegregated model based on the concept of a "dynamic specific growth rate". The predictive capability of the model has been evaluated experimentally by McLellan et al. (27). A variety of simulation tests were performed, and model predictions were found to be in reasonable agreement with experimental data. However, a more thorough analysis of the model dynamics with respect to the mathematical cause of the oscillations and the range over which periodic solutions exist has not been presented. Below we show that bifurcation analysis is well suited to answer these important questions.

Transient Model. Daugulis et al. (10) argue that many models for continuous Zymomonas mobil is cultures require measurements of physiological quantities that are difficult and time-consuming to obtain. As an alternative, they propose the concept of a "dynamic specific growth rate" which explicitly accounts for the effect of past culture conditions on subsequent cell behavior. On the basis of this concept, a simple transient model that requires only measurements of extracellular variables such as ethanol, substrate, and cell mass concentrations is proposed. The unstructured, unsegregated model consists of material balances on biomass, ethanol and substrate combined with two additional equations that describe the inhibition of cell growth caused by the past rate of change of the ethanol concentration. The model equations are

$$
\begin{aligned}
\frac{\mathrm{dX}}{\mathrm{dt}} & =[\mu(\mathrm{S}, \mathrm{P}, \mathrm{Z})-\mathrm{D}] \mathrm{X} \\
\frac{\mathrm{dS}}{\mathrm{dt}} & =-\left(\frac{1}{\mathrm{Y}_{\mathrm{p} / \mathrm{s}}}\right) \mathrm{Q}_{\mathrm{p}} \mathrm{X}+\mathrm{D}\left(\mathrm{S}_{\mathrm{f}}-\mathrm{S}\right) \\
\frac{\mathrm{dP}}{\mathrm{dt}} & =\mathrm{Q}_{\mathrm{p}} \mathrm{X}-\mathrm{DP} \\
\frac{\mathrm{dZ}}{\mathrm{dt}} & =\beta(\mathrm{W}-\mathrm{Z}) \\
\frac{\mathrm{dW}}{\mathrm{dt}} & =\beta\left(\mathrm{Q}_{\mathrm{p}} \mathrm{X}-\mathrm{DP}-\mathrm{W}\right)
\end{aligned}
$$

where $\mathrm{X}, \mathrm{S}$ and $\mathrm{P}$ are the biomass, substrate, and ethanol concentrations, respectively; $W$ and $Z$ are the first-order and second-order weighted averages, respectively, of the ethanol concentration change rate; $\mathrm{D}$ is the dilution rate; $\mathrm{S}_{\mathrm{f}}$ is the feed substrate concentration; $\mu(\mathrm{S}, \mathrm{P}, \mathrm{Z})$ is the dynamic specific growth rate; $Q_{P}$ is the specific production rate; $Y_{P / S}$ is the yield coefficient between glucose and ethanol; and $\beta$ is a parameter that determines the magnitude of the time lag for the delayed inhibition effect. More precise definitions of W, Z, $\mu$, and $Q_{p}$ can be 


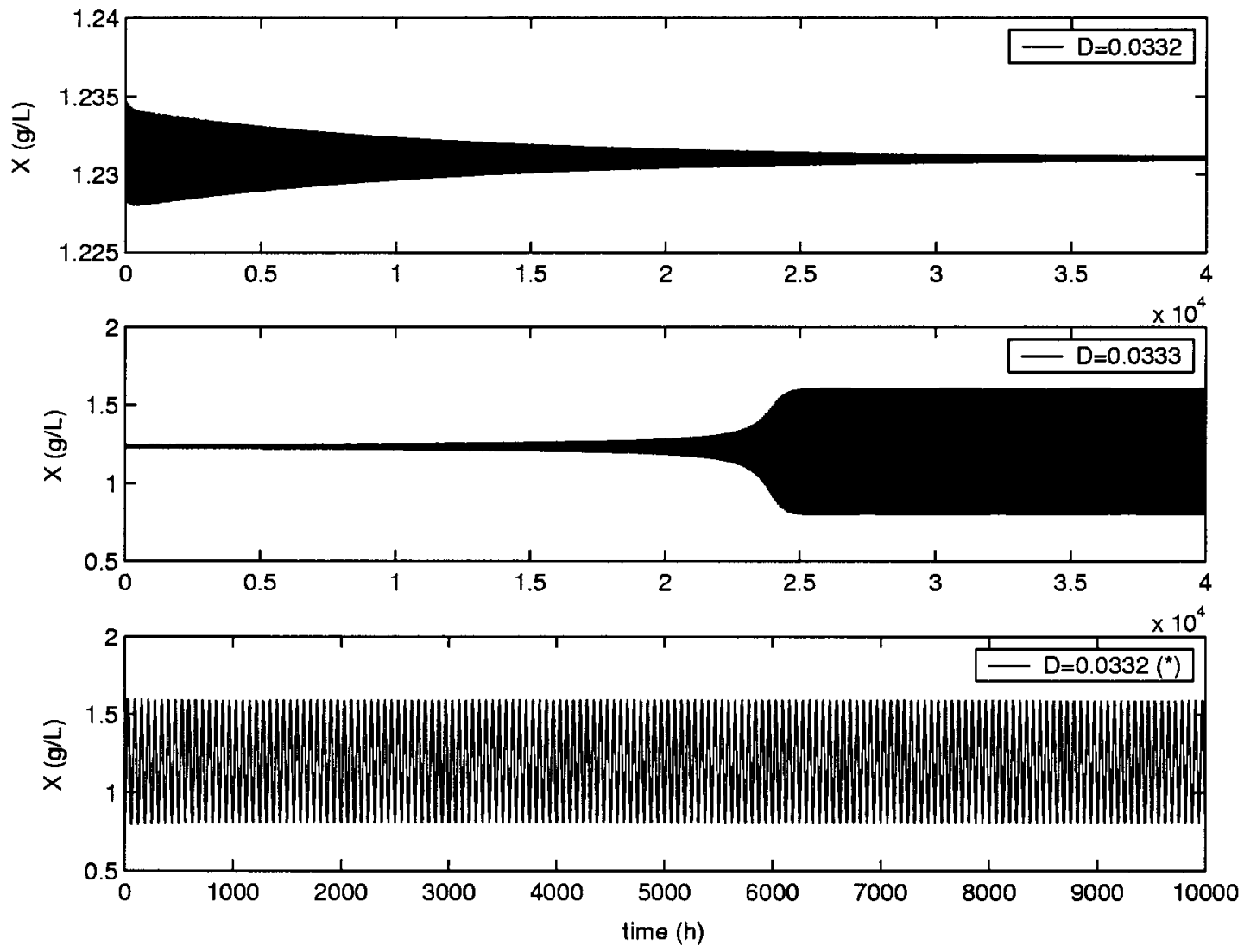

Figure 4. Dynamic simulation of the Zymomonas mobilis reactor model at low dilution rates.

found in the original reference (27). The model parameters used in the following simulation and bifurcation analysis are taken from the original reference and are listed in Table 2.

Results and Discussion. Dynamic simulations were performed to study the oscillatory behavior of the model in different regions of the operating space. Figure 3 shows two simulation tests at high dilution rates. The cell mass concentration $(X)$ is chosen as the output variable for the culture. Both simulations start at the steady state corresponding to $D=0.1120 \mathrm{~h}^{-1}$ and $S_{f}=200 \mathrm{~g} / \mathrm{L}$. The feed substrate concentration is maintained at this value and the dilution rate is changed to a slightly lower value. For a step change to $\mathrm{D}=0.1116 \mathrm{~h}^{-1}$, the culture oscillates with very small amplitude and the oscillations slowly decay to a steady-state solution that is close to the initial point. For $D=0.1115 \mathrm{~h}^{-1}$, the culture exhibits sustained oscillations of a rather small amplitude. Note that the change in dynamic behavior for the two dilution rates is not dramatic. While these tests imply that the model exhibits a bifurcation in this region, it is a time-consuming task to find by dynamic simulation the dilution rate at which this bifurcation occurs.

Figure 4 shows the results of three simulation tests at lower dilution rates and the same constant feed substrate concentration. The initial condition for the first two tests corresponds to the steady state for $D=0.033 \mathrm{~h}^{-1}$. For a step change to $D=0.0332 \mathrm{~h}^{-1}$, the culture oscillates with very small amplitude until a new steady state is reached after a very long period of time. For $D=0.0333 \mathrm{~h}^{-1}$, the culture initially oscillates with small amplitude but eventually exhibits a transition to large amplitude oscillations. In the third test, the simulation is restarted from an oscillatory initial condition from the last test $(D=$ $0.0333 \mathrm{~h}^{-1}$ ) and the dilution rate is stepped back to $\mathrm{D}=$ $0.0332 \mathrm{~h}^{-1}$. Sustained oscillations are maintained despite
Table 2. Zymomonas mobilis Reactor Model Parameters

\begin{tabular}{clll}
\hline parameter & \multicolumn{1}{c}{ value } & parameter & \multicolumn{1}{c}{ value } \\
\hline$\mu_{\max }$ & $0.41 \mathrm{~h}^{-1}$ & $\mathrm{Q}_{\mathrm{p}, \max }$ & $2.613 \mathrm{~h}^{-1}$ \\
$\mathrm{P}_{\mathrm{ob}}$ & $50.0 \mathrm{~g} / \mathrm{L}$ & $\mathrm{K}_{\mathrm{s}}$ & $0.5 \mathrm{~g} / \mathrm{L}$ \\
$\mathrm{P}_{\mathrm{ma}}$ & $217.0 \mathrm{~g} / \mathrm{L}$ & $\mathrm{K}_{\mathrm{mp}}$ & $0.5 \mathrm{~g} / \mathrm{L}$ \\
$\mathrm{P}_{\mathrm{mb}}$ & $108.0 \mathrm{~g} / \mathrm{L}$ & $\mathrm{K}_{\mathrm{i}}$ & $200.0 \mathrm{~g} / \mathrm{L}$ \\
$\mathrm{P}_{\mathrm{me}}$ & $127.0 \mathrm{~g} / \mathrm{L}$ & $\mathrm{Y}_{\mathrm{P} / \mathrm{S}}$ & $0.495 \mathrm{~g} / \mathrm{g}$ \\
$\mathrm{S}_{\mathrm{i}}$ & $80.0 \mathrm{~g} / \mathrm{L}$ & $\beta$ & $0.0366 \mathrm{~h}^{-1}$ \\
$\delta$ & 0.8241 & $\lambda$ & 21.05 \\
$\alpha$ & 8.77 & $\mathrm{a}$ & 0.3142 \\
$\mathrm{~b}$ & 1.415 & &
\end{tabular}

the fact that the same dilution rate produced a steady state in the first test.

These simulations verify that the model predicts the appearance and disappearance of sustained oscillation as observed experimentally. However, the results also raise questions about (i) the large difference in amplitudes observed when sustained oscillations are initiated at low and high dilution rates; and (ii) the two different stable solutions present at the dilution rate $\mathrm{D}=0.0332$ $\mathrm{h}^{-1}$. Another issue that warrants further investigation is the use of multiple sets of parameter values for stationary and oscillatory simulations in the original reference (27). Below we show that bifurcation analysis provides a convenient framework to address these questions.

Figure 5 shows a one-parameter bifurcation diagram of the Zymomonas mobilis model where the feed substrate concentration $\left(\mathrm{S}_{\mathrm{f}}\right)$ is held constant at $200 \mathrm{~g} / \mathrm{L}$. The dilution rate (D) is chosen as the bifurcation parameter, and the cell mass concentration $(X)$ is selected as the output variable. At low dilution rates there only is a single stable steady-state solution (-). At point 2 , a bifurcation occurs where the steady-state solution becomes unstable (---) and a periodic solution of the amplitude indicated is created. At point 1 , another 


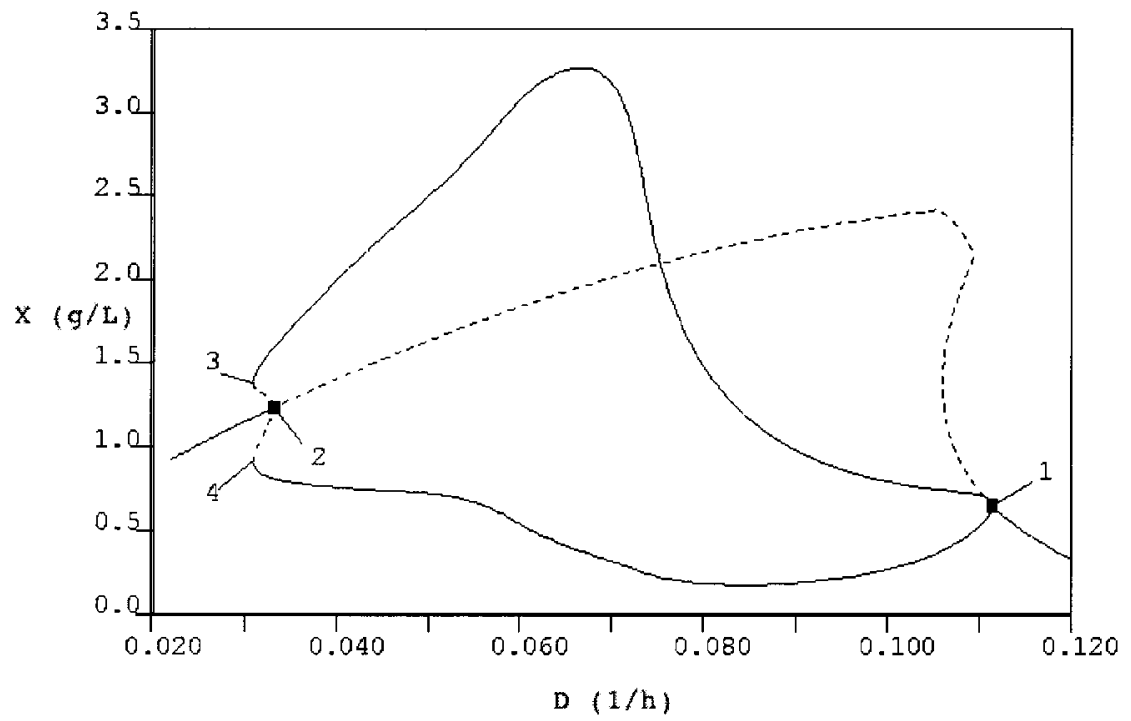

Figure 5. One-parameter bifurcation diagram of the Zymomonas mobilis reactor model.

bifurcation occurs where the periodic solution disappears and the steady-state solution becomes stable. At higher dilution rates, there is a single stable steady-state solution.

The bifurcation that occurs at point $1(D=0.11151$ $\mathrm{h}^{-1}$ ) is known as a supercritical Hopf bifurcation where loss of stability of the steady-state solution is accompanied by the appearance of very small amplitude oscillations. For the range of dilution rates between points 1 and 2 , a stable periodic solution coexists with an unstable steady-state solution. The bifurcation that occurs at point $2\left(\mathrm{D}=0.03322 \mathrm{~h}^{-1}\right)$ is known as a subcritical $\mathrm{Hopf}$ bifurcation where large amplitude oscillations appear when the steady-state sol ution becomes unstable. Points 3 and $4\left(D=0.03095 h^{-1}\right)$ represent a fold bifurcation of limit cycles. They denote the dilution rate at which the periodic solution changes stability. For dilution rates between points 2 and 3, stable and unstable periodic solutions coexist. Below point 3, the steady-state solution is the only stable solution of the model.

The one-parameter bifurcation diagram shows that the appearance and disappearance of periodic solutions is due to the existence of two Hopf bifurcation points. The analysis also answers the questions raised about the different amplitudes of sustained oscillations at low and high dilution rates. Since the Hopf bifurcation at the lower dilution rate is subcritical, stable periodic solutions are characterized by large magnitude oscillations and the stable periodic sol ution coexists with a stable steady-state solution over a very small range of dilution rates. By contrast, the Hopf bifurcation at the higher dilution rates is supercritical and the associated periodic solutions have very small amplitude.

Further characterization of the model behavior can be obtained by computing the locus of each $\mathrm{H}$ opf bifurcation point in the $D$ and $S_{f}$ plane. This is known as a two-parameter bifurcation diagram, and it allows the range of operating conditions under which periodic solutions exist to be determined. The two-parameter bifurcation diagram is shown in Figure 6. The upper branch between points 1 and 4 represents the locus of the supercritical bifurcation point, while the lower branch between points 5 and 6 is the locus of the subcritical bifurcation point. These two branches define a closed region in the $\mathrm{D}-\mathrm{S}_{\mathrm{f}}$ plane in which stable periodic solutions exist and stable steady-state solutions cannot exist. Outside this region, sustained oscillations occur over a very small range of operating conditions and coexist with stable steady-state solutions. The diagram allows concise determination of the operating conditions that support sustained oscillations and is well suited for validating the model against data. It is very difficult to obtain this type of information using only dynamic simulation.

The two-parameter bifurcation analysis also provides insights into the structural limitations of the model. In the original reference (27), three sets of model parameters were estimated to fit data when either the steady-state solution or the periodic solution was stable. The parameter values used in this study were obtained in ref 27 from oscillatory data. While each set of parameters provides a reasonable fit to the corresponding data, the lack of validation tests raises concerns about the simple model structure. In one set of experiments (27), the culture reaches a steady state for $D=0.133 \mathrm{~h}^{-1}$ and $\mathrm{S}_{\mathrm{f}}$ $=150 \mathrm{~g} / \mathrm{L}$. By contrast, the two-parameter bifurcation diagram in Figure 6 indicates that a periodic solution is expected for these values. This indicates that the model cannot be reconciled against experimental data and a more sophisticated model structure is needed. In fact, the authors (27) note that oscillatory behavior is associated with a change in cell morphology. This suggests that a segregated model may be more appropriate.

\section{Saccharomyces cerevisiae Reactor Model}

Background. Saccharomyces cerevisiae (baker's yeast) is an important microorganism in a number of industries including brewing, baking, food manufacturing, and genetic engineering. Many investigators have shown that continuous cultures of Saccharomyces cerevisiae exhibit sustained oscillations in glucose limited environments under aerobic growth conditions $(28,29,34,35)$. The underlying cellular mechanisms that cause oscillatory yeast dynamics are controversial and have been a subject of three decades of intensive research. A large number of transient models have been proposed to explain the existence of sustained oscillations $(20,34,39)$. J ones and Kompala (20) have proposed a cybernetic model that is able to predict the appearance and disappearance of sustained oscillations in continuous yeast bioreactors. A variety of simulation tests were performed to evaluate the dynamic behavior of the model. Such tests provide an incomplete characterization of the model properties that is biased toward the specific behaviors being investigated. Below we show that a detailed bifurcation 


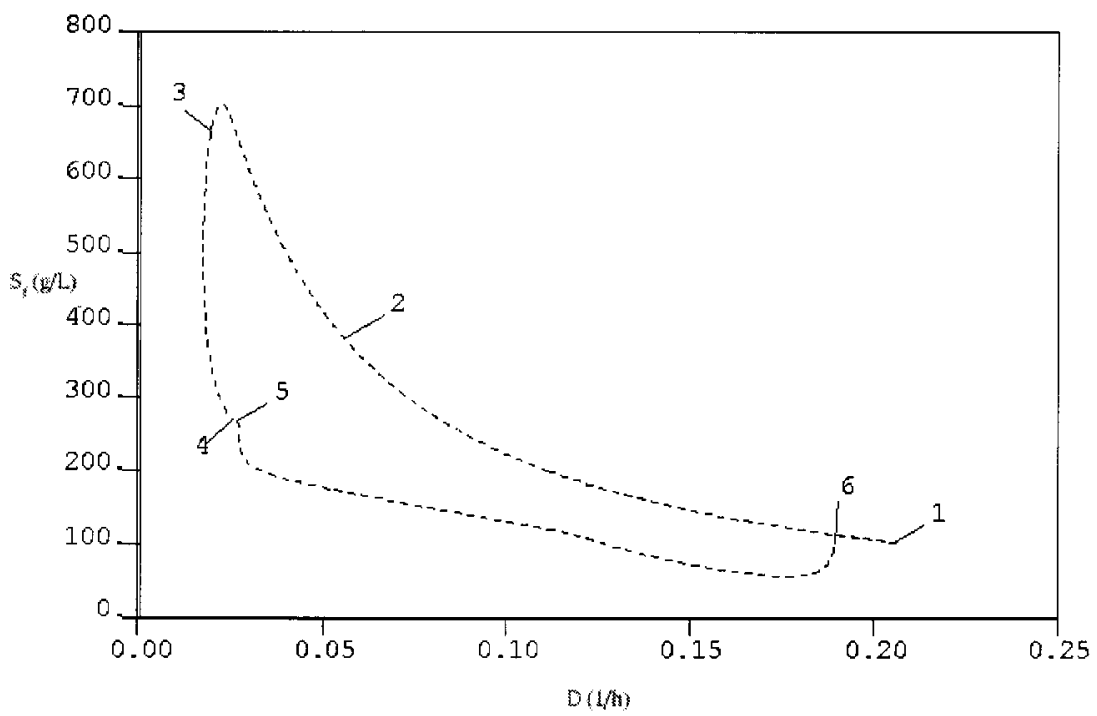

Figure 6. Two-parameter bifurcation diagram of the Zymomonas mobilis reactor model.

analysis can reveal complex dynamic behavior that is unlikely to be discovered using dynamic simulation alone.

Transient Model. On the basis of the cybernetic modeling framework (32), J ones and Kompala (20) have developed a structured, unsegregated model for continuous yeast bi oreactors. Sustained oscillations are viewed as the result of competition between three metabolic pathways: glucose fermentation, ethanol oxidation, and glucose oxidation. Detailed modeling of the intracellular regulatory processes is replaced by cybernetic variables $u_{i}$ and $v_{i}$ representing the optimal strategies for enzyme synthesis and activity, respectively. Denoting the instantaneous growth rate along the i-th pathways as $r_{i}$, the optimal strategies for $u_{i}$ and $v_{i}$ are

$$
\begin{aligned}
& \mathrm{u}_{\mathrm{i}}=\frac{\mathrm{r}_{\mathrm{i}}}{\sum_{\mathrm{j}} \mathrm{r}_{\mathrm{j}}} \\
& \mathrm{v}_{\mathrm{i}}=\frac{\mathrm{r}_{\mathrm{i}}}{\max _{\mathrm{j}} \mathrm{r}_{\mathrm{j}}}
\end{aligned}
$$

If the growth rate along the i-th pathway is large, then the associated synthesis $\left(u_{i}\right)$ and activity $\left(v_{i}\right)$ will be large. The growth rate $r_{i}$ al ong each pathway is modeled with modified Monod rate equations in which the rate is proportional to the intracellular concentration of a key enzyme e controlling the i-th pathway:

$$
\begin{aligned}
& r_{1}=\mu_{1} e_{1} \frac{G}{K_{1}+G} \\
& r_{2}=\mu_{2} e_{2} \frac{E}{K_{2}+E} \frac{O}{K_{O_{2}}+O} \\
& r_{3}=\mu_{3} e_{3} \frac{G}{K_{3}+G} \frac{O}{K_{O_{3}}+O}
\end{aligned}
$$

where $\mathrm{G}, \mathrm{E}$, and $\mathrm{O}$ represent the concentrations of glucose, ethanol and dissolved oxygen, respectively; $\mu_{\mathrm{i}}$ are maximum growth rate constants; $\mathrm{K}_{\mathrm{i}}$ and $\mathrm{K}_{\mathrm{O}_{i}}$ are saturation constants for the substrate and dissolved oxygen, respectively.

The cybernetic model equations presented in the original reference (20) contain several typographical errors. The corrected mass balances are written as:

$$
\begin{aligned}
& \frac{d X}{d t}=\left(\sum_{i}\left(r_{i} v_{i}\right)-D\right) X \\
& \frac{d C}{d t}=\gamma_{3} r_{3} v_{3}-\left(\gamma_{1} r_{1} v_{1}+\gamma_{2} r_{2} v_{2}\right) C-\sum_{i}\left(r_{i} v_{i}\right) C \\
& \frac{d G}{d t}=D\left(G_{0}-G\right)-\left(\frac{r_{1} v_{1}}{Y_{1}}+\frac{r_{3} v_{3}}{Y_{3}}\right) X-\phi_{4}\left(C \frac{d X}{d t}+X \frac{d C}{d t}\right) \\
& \frac{d E}{d t}=-D E+\left(\phi_{1} \frac{r_{1} v_{1}}{Y_{1}}-\frac{r_{2} v_{2}}{Y_{2}}\right) X \\
& \frac{d O}{d t}=k_{L} a(O *-O)-\left(\phi_{2} \frac{r_{2} v_{2}}{Y_{2}}+\phi_{3} \frac{r_{3} v_{3}}{Y_{3}}\right) X \\
& \frac{d e_{1}}{d t}=\alpha u_{1} \frac{G}{K_{1}+G}-\left(\sum_{i}\left(r_{i} v_{i}\right)+\beta\right) e_{1}+\alpha^{*} \\
& \frac{d e_{2}}{d t}=\alpha u_{2} \frac{E}{K_{2}+E}-\left(\sum_{i}\left(r_{i} v_{i}\right)+\beta\right) e_{2}+\alpha^{*} \\
& \frac{d e_{3}}{d t}=\alpha u_{3} \frac{G}{K_{3}+G}-\left(\sum_{i}\left(r_{i} v_{i}\right)+\beta\right) e_{3}+\alpha^{*}
\end{aligned}
$$

where $X$ and $C$ are the cell mass concentration and intracellular storage carbohydrate mass fraction, respectively; $D$ is the dilution rate; $G_{0}$ is the glucose feed concentration; $\mathrm{k}_{\mathrm{L}} \mathrm{a}$ is the dissolved oxygen mass transfer coefficient; $\mathrm{O}^{*}$ is the saturation concentration of oxygen; $Y_{i}$ are yield coefficients; $\phi_{i}$ and $\gamma_{i}$ are stoichiometric coefficients for substrate and intracellular storage carbohydrate synthesis and consumption, respectively; $\alpha$ and $\beta$ are enzyme synthesis and decay rate constants, re spectively; and $\alpha^{*}$ is a constant parameter associated with constitutive enzyme synthesis. A more detailed description of the model can be found in ref 20 . It is worth noting that the cybernetic model does not generate sustained oscillations with the parameter values exactly as given in ref 20 . The parameter values used in the 


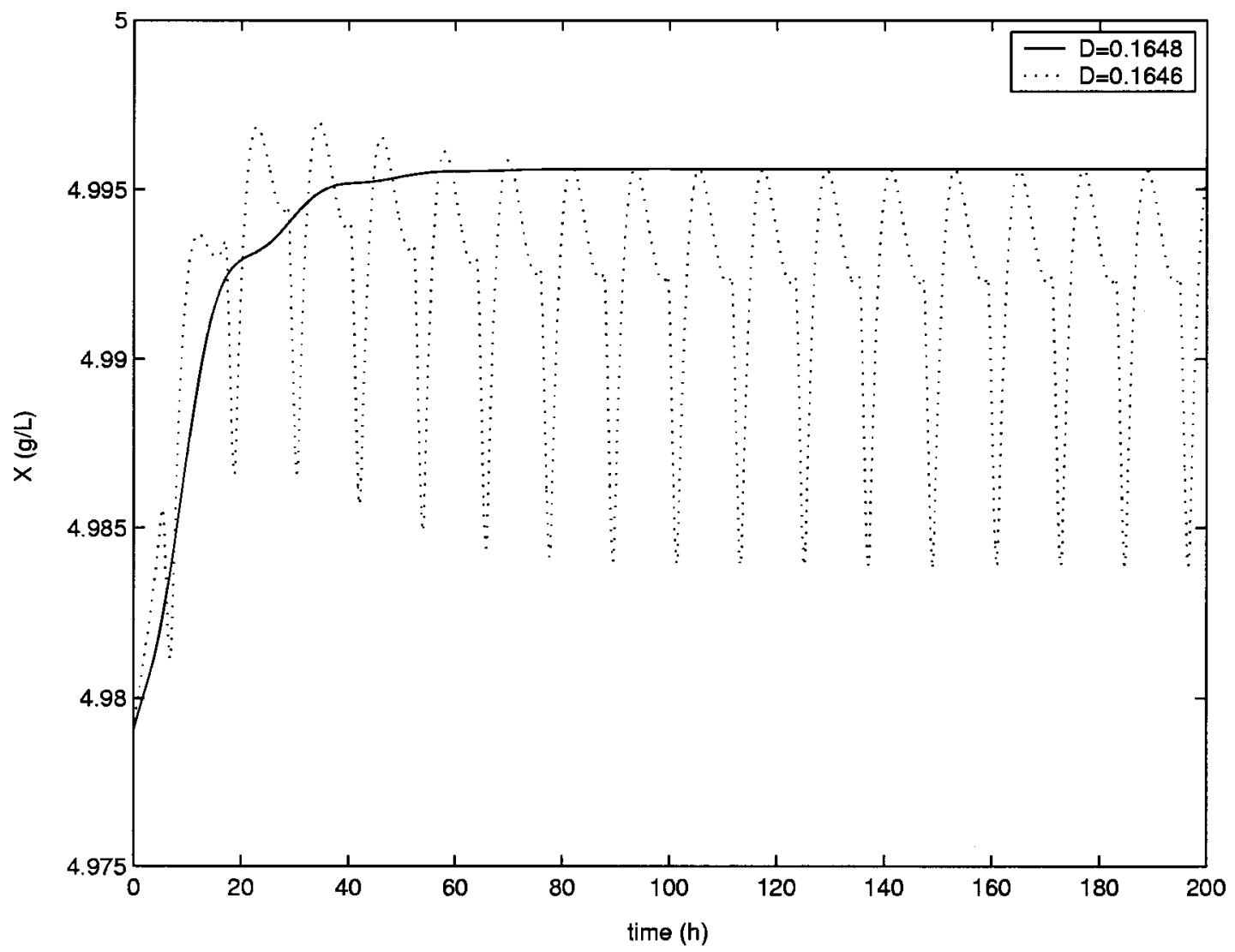

Figure 7. Dynamic simulation of the Saccharomyces cerevisiae reactor model at high dilution rates.

subsequent simulation and bifurcation studies are given in Table 3. The parameters are obtained from ref 20 with the exception that $\alpha^{*}=0.03 \mathrm{~g} / \mathrm{g}$-h.

Results and Discussion. As mentioned previously, the cybernetic model does not produce sustained oscillations with the parameter values given exactly as in ref 20. Because the value of the parameter $\alpha^{*}$ is not specified in the original reference, a primitive search was conducted to determine combinations of the parameters that support periodic solutions. The following combinations of $D$ and $G_{0}$ were found by dynamic simulation to produce sustained oscillations when $\alpha^{*}=0.03 \mathrm{~g} / \mathrm{g}-\mathrm{h}$ :

$$
\begin{aligned}
& \cdot \mathrm{D}=0.16 \mathrm{~h}^{-1} \text { and } \mathrm{G}_{0} \in[9.5 \mathrm{~g} / \mathrm{L}, 10.5 \mathrm{~g} / \mathrm{L}] \\
& \text { - } \mathrm{D}=0.14 \mathrm{~h}^{-1} \text { and } \mathrm{G}_{0} \in[9.7 \mathrm{~g} / \mathrm{L}, 12.3 \mathrm{~g} / \mathrm{L}] \\
& \text { - } \mathrm{G}_{0}=10 \mathrm{~g} / \mathrm{L} \text { and } \mathrm{D} \in\left[0.14 \mathrm{~h}^{-1}, 0.16 \mathrm{~h}^{-1}\right] \\
& \text { - } \mathrm{G}_{0}=12.3 \mathrm{~g} / \mathrm{L} \text { and } \mathrm{D} \in\left[0.118 \mathrm{~h}^{-1}, 0.14 \mathrm{~h}^{-1}\right]
\end{aligned}
$$

Clearly this is a very inefficient method for determining the range of parameter values. Below we show that bifurcation analysis is a much more powerful tool for determining such information. It is worth noting that in the original reference (20) bifurcation analysis is used to determine the range of four unknown parameters $\left(\mu_{3}\right.$ $\mathrm{K}_{3}, \mathrm{~K}_{\mathrm{O}_{2}}, \mathrm{~K}_{\mathrm{O}_{3}}$ ) that support periodic solutions.

First the transient behavior of the model is studied via dynamic simulation. Figure 7 shows two simulation tests at high dilution rates. The cell mass concentration $(X)$ is chosen as the output variable for the culture. Both simulations are initiated with the steady-state solution corresponding to $D=0.165 \mathrm{~h}^{-1}$ and $\mathrm{G}_{0}=10 \mathrm{~g} / \mathrm{L}$. The
Table 3. Saccharomyces cerevisiae Reactor Model Parameters

\begin{tabular}{cll}
\hline parameter & unit & value \\
\hline$\mu_{\mathrm{i}, \max }$ & $\mathrm{h}^{-1}$ & $0.44,0.19,0.36$ \\
$\mathrm{~K}_{\mathrm{i}}$ & $\mathrm{g} / \mathrm{L}$ & $0.05,0.01,0.001$ \\
$\mathrm{Y}_{\mathrm{i}}$ & $\mathrm{g} / \mathrm{g}$ & $0.16,0.75,0.60$ \\
$\phi_{\mathrm{i}}$ & $\mathrm{g} / \mathrm{g}$ & $0.403,2.0,1.0,0.95$ \\
$\gamma_{\mathrm{i}}$ & $\mathrm{g} / \mathrm{g}$ & $10,10,0.8$ \\
$\alpha$ & $\mathrm{g} / \mathrm{g}-\mathrm{h}$ & 0.3 \\
$\alpha^{*}$ & $\mathrm{~g} / \mathrm{g}-\mathrm{h}$ & 0.03 \\
$\beta$ & $\mathrm{h}-1$ & 0.7 \\
$\mathrm{~K}_{\mathrm{O}_{2}}$ & $\mathrm{mg} / \mathrm{L}$ & 0.01 \\
$\mathrm{~K}_{\mathrm{O}_{3}}$ & $\mathrm{mg} / \mathrm{L}$ & 2.2 \\
$\mathrm{~K}_{\mathrm{L}} \mathrm{a}$ & $\mathrm{h}-1$ & 225 \\
$\mathrm{O}^{*}$ & $\mathrm{mg} / \mathrm{L}$ & 7.5
\end{tabular}

feed glucose concentration is maintained at this value while the dilution rate is changed slightly. When the dilution rate is changed to $\mathrm{D}=0.1648 \mathrm{~h}^{-1}$ the model moves to a new steady-state solution, while for $D=$ $0.1646 \mathrm{~h}^{-1}$ the model exhibits sustained oscillations of a small amplitude. Figure 8 shows some simulation results at lower dilution rates and the same feed glucose concentration. The simulations are initiated with the steady-state solution for $D=0.13 \mathrm{~h}^{-1}$. When the dilution rate is changed to $D=0.1366 \mathrm{~h}^{-1}$, the model initially oscillates and then reaches a new steady state at approximately $200 \mathrm{~h}$. When $\mathrm{D}=0.1367 \mathrm{~h}^{-1}$, the model exhi bits sustained oscillations with a considerably larger amplitude than that shown in Figure 7. At $t=200 \mathrm{~h}$, the dilution rate of the latter simulation is reduced to $D$ $=0.1366 \mathrm{~h}^{-1}$. While the amplitude is decreased, sustained oscillations are maintained even though the same dilution rate previously produced a steady-state solution.

These simulation results demonstrate the cybernetic model is capable of predicting the appearance and 


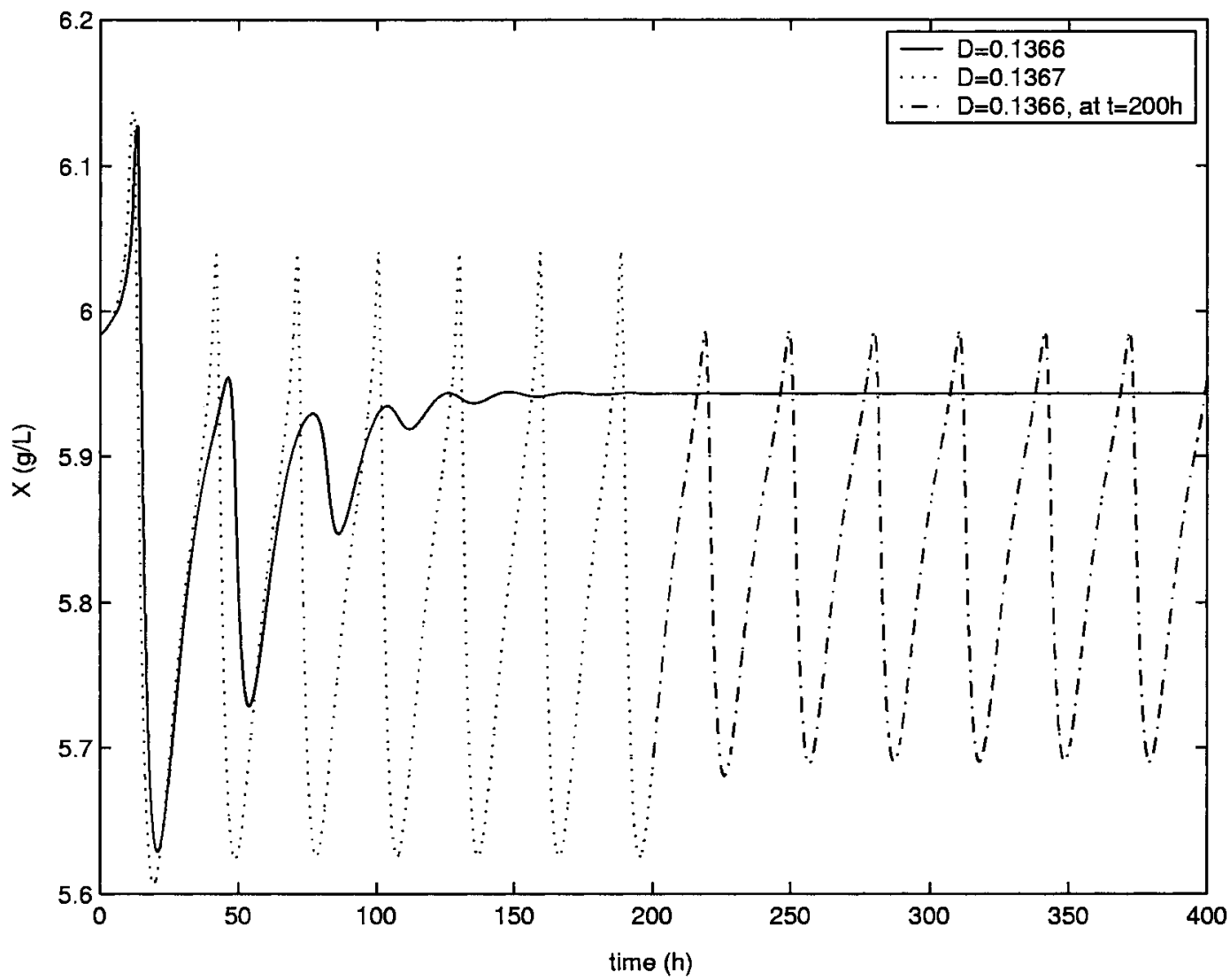

Figure 8. Dynamic simulation of the Saccharomyces cerevisiae reactor model at low dilution rates.

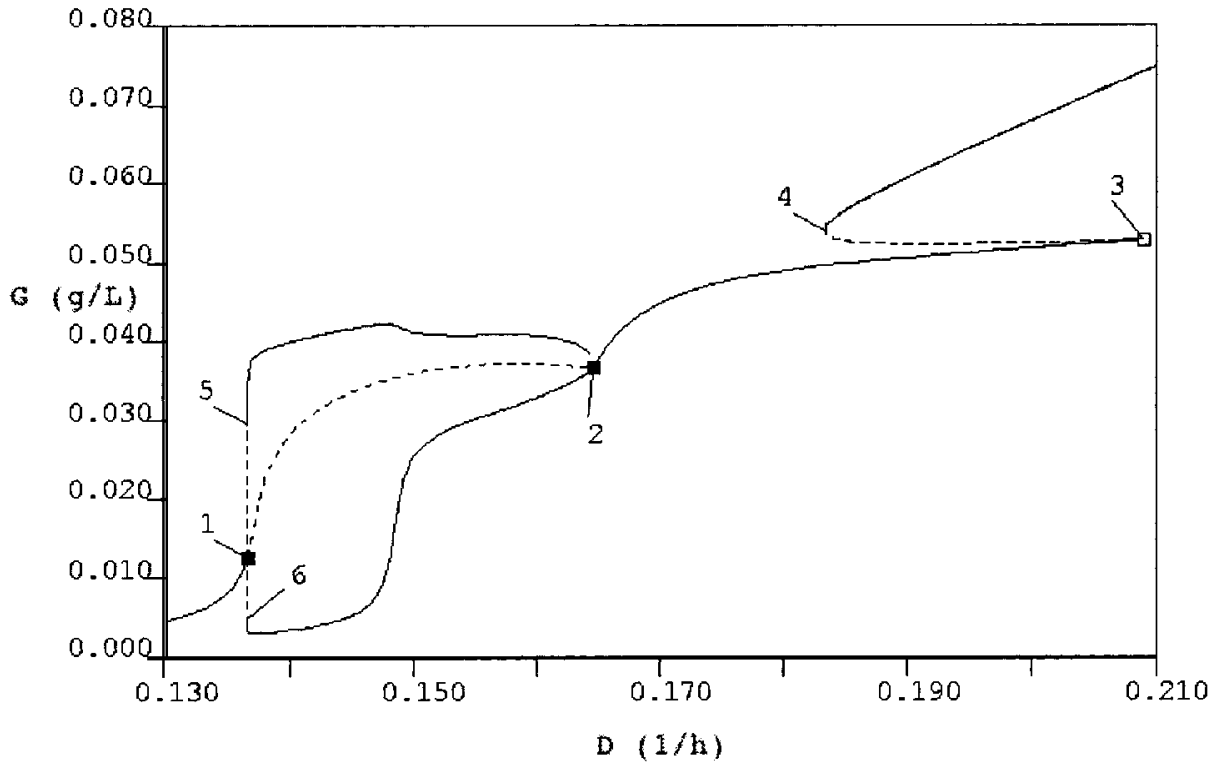

Figure 9. One-parameter bifurcation diagram of the Saccharomyces cerevisiae reactor model with $\mathrm{D}$ as the bifurcation parameter and $\mathrm{G}_{0}=10 \mathrm{~g} / \mathrm{L}$.

disappearance of sustained oscillation over a range of operating parameters. However, the results indicate significant differences between experiment and simulation with respect to the oscillation amplitude at low and high dilution rates as well as the hysteresis behavior observed at low dilution rates. We propose to investigate these issues via bifurcation analysis. It is well-known that continuous yeast bioreactors exhibit sustained oscillations only for a specific range of dilution rates (29). This suggests that the dilution rate (D) is a reasonable choice for the bifurcation parameter. In this case, the feed glucose concentration $\mathrm{G}_{0}$ is fixed at $10 \mathrm{~g} / \mathrm{L}$.
The one-parameter bifurcation diagram with $\mathrm{D}$ as the bifurcation parameter is shown in Figure 9 where the glucose concentration $(G)$ is chosen as the output variable. The solid lines represent stable steady-state and periodic solutions, and the dashed lines represent unstable solutions. The middle branch between points 1-4 represents steady-state solutions for different dilution rates. The upper branch defined by points 1,5 , and 2 represents the largest $\mathrm{G}$ value obtained for the periodic solution at the given dilution rate. The lower branch consisting of points 1,6 , and 2 defines the lower $\mathrm{G}$ limit of the periodic solutions. There is a single stable steady- 


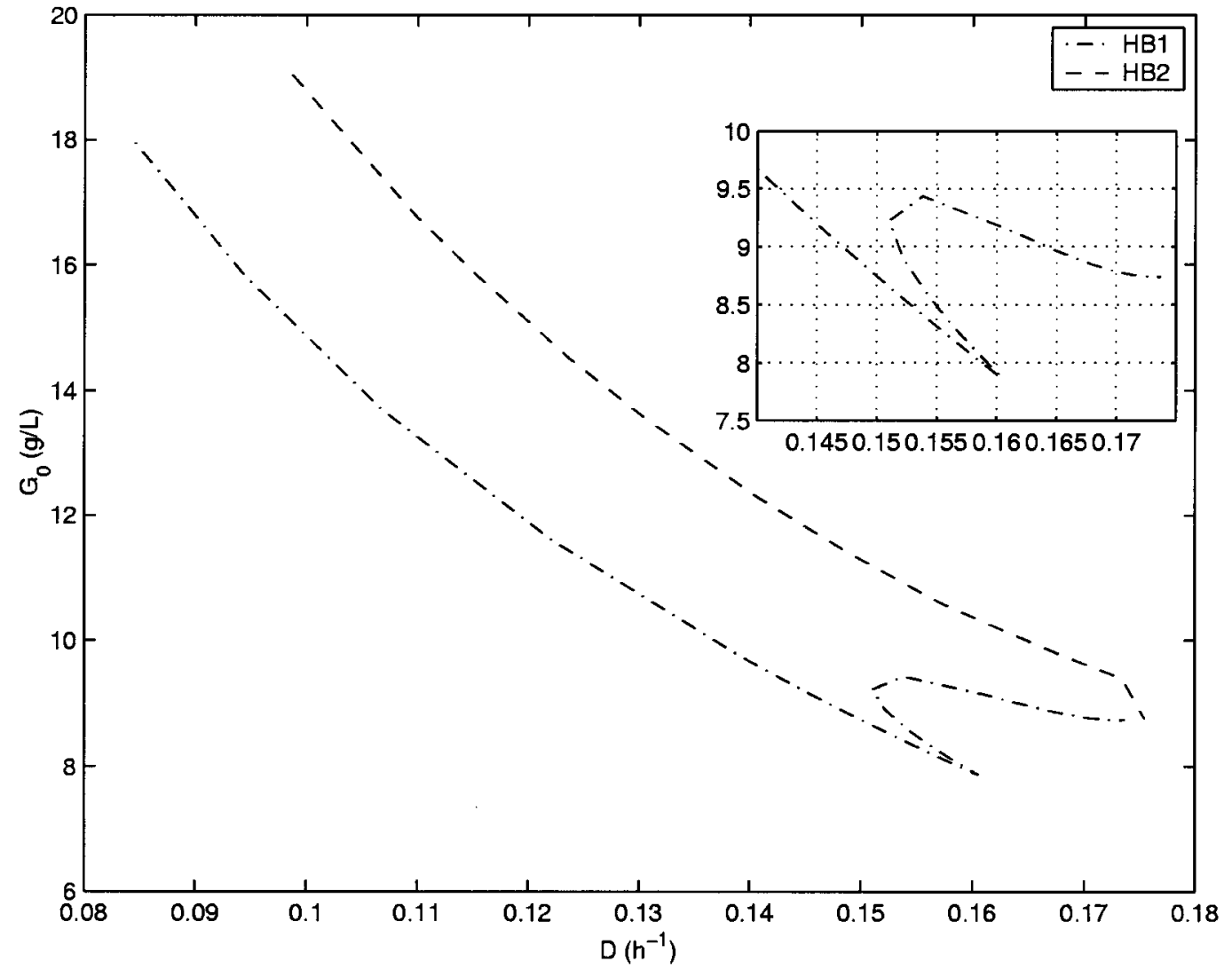

Figure 10. Two-parameter bifurcation diagram of the Saccharomyces cerevisiae reactor model with $\mathrm{D}$ and $\mathrm{G}_{0}$ as the bifurcation parameters.

state sol ution at low dilution rates. When $\mathrm{D}$ is increased to $0.13664 \mathrm{~h}^{-1}$ (point 1), a subcritical Hopf bifurcation occurs and the steady-state solution becomes unstable. The periodic solution generated initially is unstable and bends back toward low dilution rates until a fold bifurcation occurs at $D=0.13654 \mathrm{~h}^{-1}$ (point 5). Past this point the periodic solution is stable.

For a wide range of dilution rates, the stable periodic solution coexists with an unstable steady-state solution. When $D$ is increased to $0.16496 \mathrm{~h}^{-1}$ (point 2), a supercritical Hopf bifurcation occurs, which causes the periodic solution to disappear and the steady-state solution to regain its stability. The steady-state solution maintains its stability until a branching point (22) at $D=0.20901$ $\mathrm{h}^{-1}$ (point 3 ) is reached. Then the steady-state solution turns back in the direction of decreasing dilution rate and become unstable. Another fold bifurcation occurs at $\mathrm{D}=0.18341 \mathrm{~h}^{-1}$ (point 4) where the steady-state solution becomes stable once again. Although not shown, higher dilution rates will cause the steady state to lose stability and the wash-out steady state to become stable.

The bifurcation structure shown in Figure 9 provides a concise explanation of the previous dynamic simulation results. The appearance and disappearance of sustained oscillations is attributable to two Hopf bifurcations, as also was noted by J ones et al. (19). However, considerably more information can be extracted from the bifurcation diagram:

- The first Hopf bifurcation is subcritical, and the associated periodic solution undergoes a fold bifurcation. Consequently, there exists a very small range of dilution rates $\left[0.13654 \mathrm{~h}^{-1}, 0.13664 \mathrm{~h}^{-1}\right]$ where there coexists a stable steady state, an unstable periodic solution, and a stable periodic solution. For these parameter values, the range is too small for the multiple stable solutions to be observed experimentally. Nevertheless, the subcritical form of the bifurcation agrees with the experimental observation, and the simulation results (Figure 8) that sustained oscillations at lower dilution rates have a large amplitude once established.

- The second Hopf bifurcation point is supercritical. Therefore, the periodic solution that emanates from this bifurcation has very small amplitude. This explains the dynamic simulation results in Figure 7. To our knowledge there are no experimental studies that demonstrate a difference between the oscillation amplitudes that arise from bifurcations at low and high dilution rates. However, this model prediction could be investigated experimentally.

- There is hysteresis for dilution rates in the range of $\mathrm{D} \in\left[0.18341 \mathrm{~h}^{-1}, 0.20901 \mathrm{~h}^{-1}\right]$ due to the presence of multiple stable steady-state solutions. Hysteresis could have an adverse effect on the reactor operability. It appears that such behavior has not been reported by other theoreticians or experimentalists; further experimental studies are required to test this model prediction.

Additional knowledge can be obtained by investigating the bifurcation behavior with respect to two parameters simultaneously. Figure 10 shows the two-parameter bifurcation diagram with dilution rate (D) and feed glucose concentration $\left(\mathrm{G}_{0}\right)$ as the bifurcation parameters. The figure depicts the locus of the Hopf bifurcation points where HB1 and HB2 refer to points 1 and 2, respectively, in Figure 9. The region contained inside these two loci represents the operating space that supports sustained oscillations. Unlike the two-parameter bifurcation diagram for the Zymomonas mobilis model in Figure 6, the parameter region is not closed and there is a multiplicity phenomena as shown in the inset. The locus for HB2 is monotonic, whilethe locus for HB1 has an "S" shape. This 


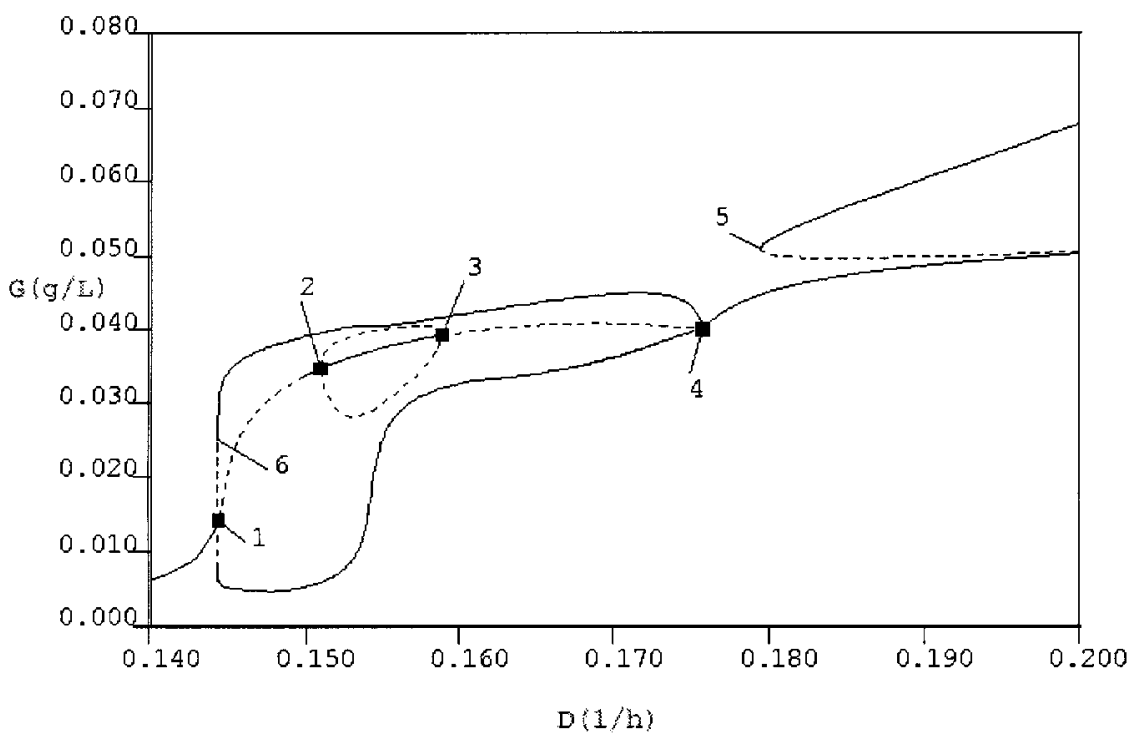

Figure 11. One-parameter bifurcation diagram of the Saccharomyces cerevisiae reactor model with $\mathrm{D}$ as the bifurcation parameter and $\mathrm{G}_{0}=9.25 \mathrm{~g} / \mathrm{L}$.

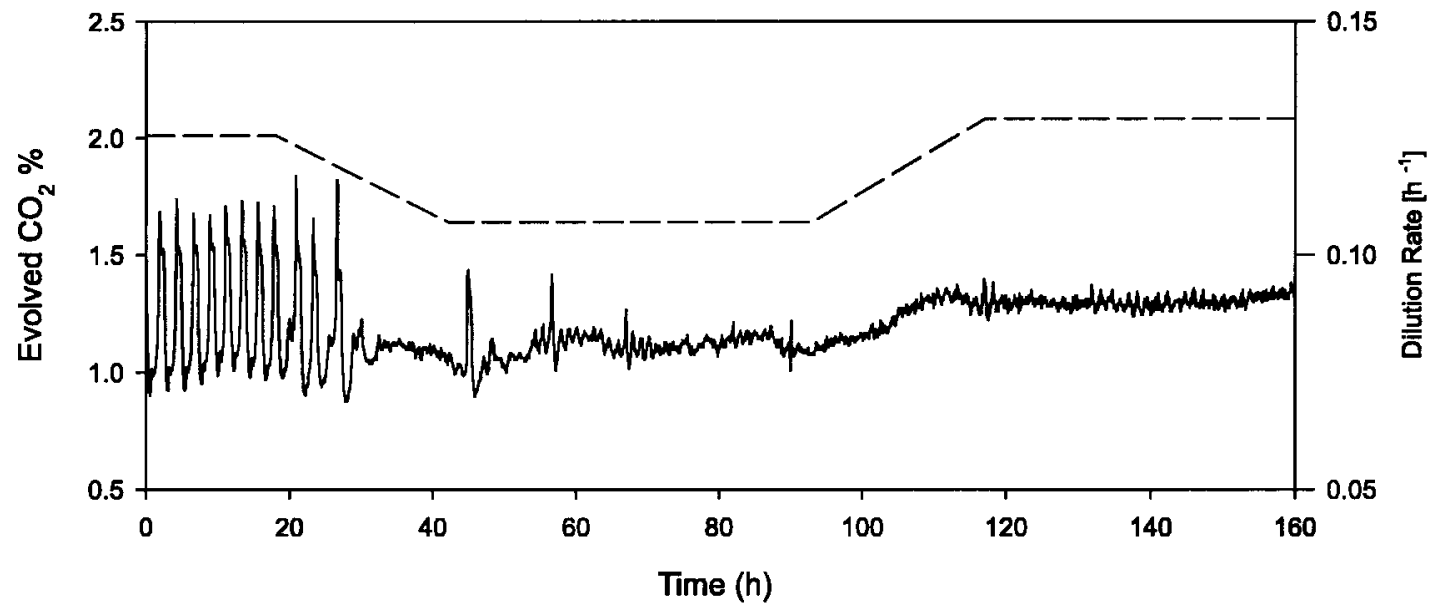

Figure 12. Experimentally observed transient behavior in a continuous culture of Saccharomyces cerevisiae for ramp changes in the dilution rate.

implies that for a certain region of operating conditions, HB1 bifurcates into multiple bifurcation points at a fixed $\mathrm{G}_{0}$ or a fixed $\mathrm{D}$. Therefore, a one-parameter bifurcation analysis with the other parameter fixed at an appropriate value will yield two or three bifurcation points that originate from point 1 in Figure 9. As shown in Figure 10 , there is a single bifurcation point for $\mathrm{G}_{0}=10 \mathrm{~g} / \mathrm{L}$.

The one-parameter bifurcation diagram with $\mathrm{D}$ as the bifurcation parameter and $\mathrm{G}_{0}=9.25 \mathrm{~g} / \mathrm{L}$ is shown in Figure 11. Similar to Figure 9, the branch between points 1-4 represents a steady-state solution contained within a periodic solution appearing from the subcritical $\mathrm{Hopf}$ bifurcation point (point 1 ) and disappearing at the supercritical Hopf bifurcation (point 4). The periodic solution emanating from point 1 is unstable until the fold bifurcation (point 6).

Unlike Figure 9, there now exists an unstable periodic solution within the large-amplitude stable periodic solution. The unstable periodic solution emanates from a supercritical Hopf bifurcation (point 2) and ends at another supercritical Hopf bifurcation (point 3). Within this periodic solution, the steady-state solution regains its stability. Therefore, within the range $D \in[0.15100$ $\mathrm{h}^{-1}, 0.15883 \mathrm{~h}^{-1}$ ] there is a stable steady-state solution, an unstable periodic solution, and a stable periodic solution. The cybernetic model can predict the coexistence of a stable steady-state solution and a stable periodic solution over a meaningful range of dilution rates. It is important to emphasize that the existence of multiple attractors would be quite difficult to observe using dynamic simulation alone. This again supports our contention that bifurcation analysis is a very powerful tool for model analysis and validation.

The behavior shown in Figure 11 is particularly interesting as we have observed experimentally multiple attractors in continuous cultures of Saccharomyces cerevisiae(37). Figure 12 shows the results of an experiment designed to examine this phenomenon. The evolved carbon dioxide signal is used as a representative output variable for the culture. The experiment starts with oscillatory dynamics that are obtained by switching the culture from batch to continuous operation. At $\mathrm{t}=20 \mathrm{~h}$ the dilution rate is slowly ramped down over a $24 \mathrm{~h}$ period until the oscillations disappear. The stationary state is preserved for 2 days while the dilution rate is maintained at the low value. At $\mathrm{t}=92 \mathrm{~h}$ the dilution rate is slowly ramped up at the same rate as was used for the negative ramp. Oscillations are not observed when the dilution rate is maintained at the high value despite the fact that a slightly lower dilution rate produced oscillations at the beginning of the experiment. An enlarged view of the dynamic behavior during the first 

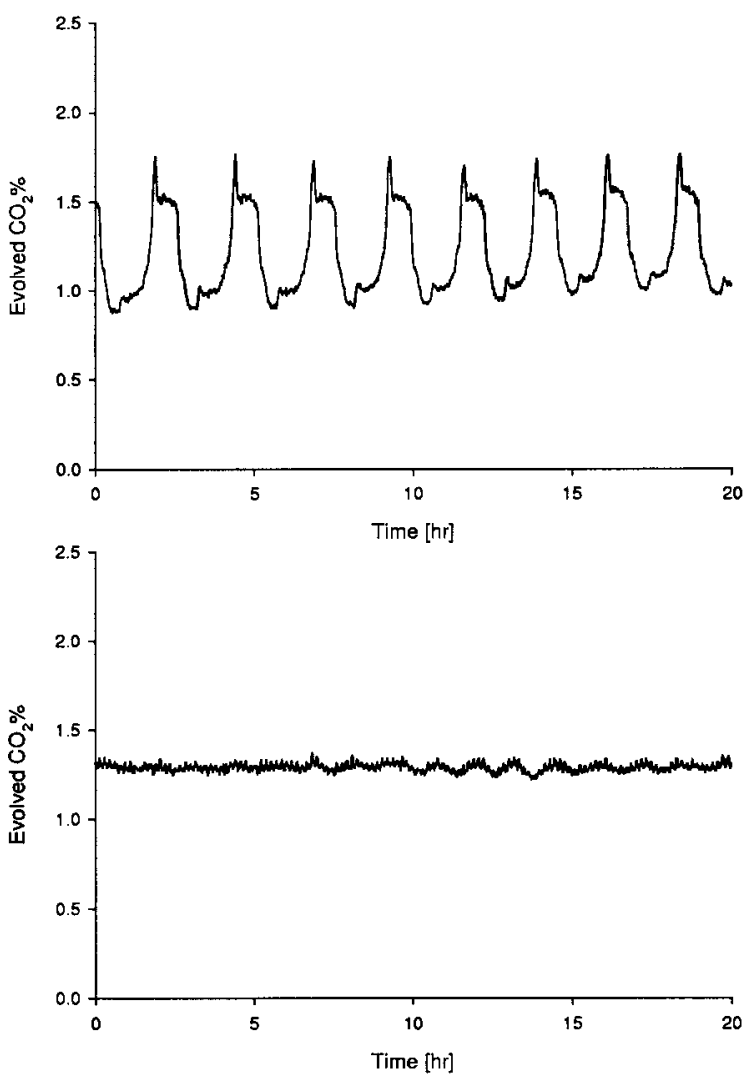

Figure 13. Experimentally observed multiple attractors in a continuous culture of Saccharomyces cerevisiae

and last parts of the experiment is shown in Figure 13. The upper plot shows the large amplitude oscillations obtained at the beginning of the experiment, while the lower plot shows the stationary response observed at the end of the experiment. The bifurcation analysis in Figure 11 suggests that the cybernetic model can predict the existence of such multiple attractors.

A one-parameter bifurcation diagram with $D$ as the bifurcation parameter and $\mathrm{G}_{0}$ fixed at $8.7 \mathrm{~g} / \mathrm{L}$ is presented in Figure 14. As shown in Figure 10, there are two bifurcation points that emanate from point 1 in Figure
9. In this case, both of the Hopf bifurcations are subcritical. The range of dilution rates that support periodic solution is quite small, and sustained oscillations might be difficult to observe experimentally. The bifurcation structure for dilution rates outside the region shown is very similar to that in Figure 9 and has been omitted.

\section{Summary and Conclusions}

We have studied the dynamic behavior of three continuous bioreactor models that exhibit complex steadystate and transient behavior. Bifurcation analysis is shown to provide a more complete picture of model behavior than is possible with dynamic simulation alone. The determination of bifurcation points when the qualitative model behavior changes and the characterization of the range of parameter values that supports certain behaviors is valuable information for model validation. Several important characteristics of the three models studied that are not observed in previous simulation studies are revealed through bifurcation analysis. These characteristics include lack of model robustness to small parameter variations, apparent inconsistencies between model structure and experimental data, and the coexistence of multiple stable solutions under the same operating conditions. These case studies suggests that bifurcation analysis is a very powerful tool for analyzing lowdimensional bioreactor models and should be used in conjunction with dynamic simulation for model validation.

\section{Acknowledgment}

Financial support from the LSU Department of Chemical Engineering is gratefully acknowledged. The authors would like to acknowledge Prof. Martin Hjortso for his assistance in acquiring the Saccharomyces cerevisiae experimental data and Prof. E usebius Doedel (Concordia University) for providing assistance with the continuation package AUTO. The authors also would like to acknowledge Prof. Doraiswami Ramkrishna (Purdue) and Dhinakar Kompala (Colorado) for their assistance with the Hybridoma and Saccharomyces cer evisae reactor models, respectively.

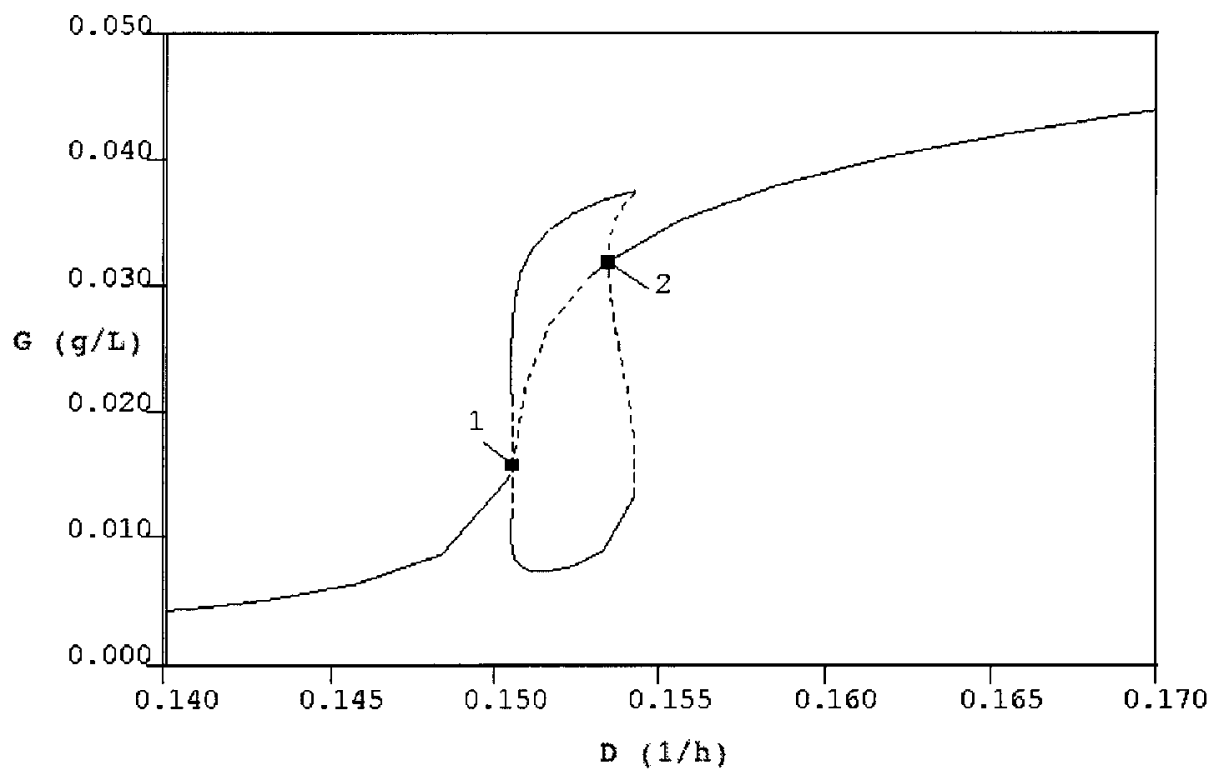

Figure 14. One-parameter bifurcation diagram of the Saccharomyces cerevisiae reactor model with $\mathrm{D}$ as the bifurcation parameter and $\mathrm{G}_{0}=8.75 \mathrm{~g} / \mathrm{L}$ 


\section{References and Notes}

(1) Ajbar, A. On the existence of oscillatory behavior in unstructured model of bioreactors. Chem. Eng. Sci. 2001, 56, 1991-1997.

(2) Ajbar, A.; Alhumazi, K. Microbial competition: Study of global branching phenomena. AIChE J . 2000, 46, 321-334.

(3) Ajbar, A.; Ibrahim, G. Stability and bifurcation of an unstructured model of bioreactor with cell recycle. Math. Comput. Modeling 1997, 25, 9-30.

(4) Baltzis, B. C.; Fredrickson, A. G. Limitation of growth rate by two complementary nutrients: Some elementary but neglected considerations. Biotechnol. Bi oeng. 1988, 31, 7586.

(5) Beavan, M. J . Charpentier, C.; Rose, A. H. Production and tolerance of ethanol in relation to phospholipid fatty-acyl composition in Saccharomyces cerevisiae NCY C 431. J . Gen. Microbiol. 1982, 128, 1447-1455.

(6) Bellgardt, K.-H. Analysis of synchronous growth of baker's yeast: Part I: Development of a theoretical model for sustained oscillations. J . Biotechnol. 1994, 35, 19-33.

(7) Bruce, L. J .; Axford, D. B.; Ciszek, B.; Daugulis, A. J . Extractive fermentation by Zymomonas mobilis and the control of oscillatory behavior. Biotechnol. Lett. 1991, 128, 291-296.

(8) Cazzador, L. Analysis of oscillations in yeast continuous cultures by a new simplified model. Bull. Math. Biol. 1991 $53,685-700$

(9) Cazzador, L.; Alberghina, L.; Martegani, E.; Mariani, L. Bioreactor control and modeling: A simulation program based on a structured population model of budding yeast. In Bioreactors and Biotransformati ons; M oody, G. W., Baker, P. B., Eds.; Elsevier: London, 1987; pp 64-75.

(10) Daugulis, A. J .; McLellan, P. J.; Li, J. Experimental investigation and modeling of oscillatory behavior in the continuous culture of Zymomonas mobilis. Bi otechnol. Bioeng. 1997, 56, 99-105.

(11) Doedel, E.; Wang, X.; Fairgrieve, T. AUTO: Software for continuation and bifurcation problems in ordinary differential equations. Technical report, California Institute of Technology, 1986.

(12) Duboc, P.; von Stockar, U. Modeling of oscillating cultivations of Saccharomyces cerevisiae: I dentification of population structure and expansion kinetics based on on-line measure ments. Chem. Eng. Sci. 2000, 55, 149-160.

(13) Eakman, J. M.; Fredrickson, A. G.; Tsuchiya, H. H. Statistics and dynamics of microbial cell populations. Chem. Eng. Prog. Symp. Ser. 1966, 62, 37-49.

(14) Gambhir, A.; Europa, A. F.; Hu, W. S. Alteration of cellular cetabolism by consecutive fed-batch cultures of mammalian cells. J . Biosci. Bioeng. 1999, 87, 805-810.

(15) Ghommidh, C.; Vaija, J .; Bolarinwa, S.; Navarro, J . M. Oscillatory behavior of Zymomonas mobilis in continuous cultures: A simple stochastic model. Biotechnol. Lett. 1989, $11,659-664$.

(16) Guardia, M. J .; Gambhir, A.; Europa, A. F.; Ramkrishna, D.; Hu, W. S. Cybernetic modeling and regulation of metabolic pathways in multiple steady states of Hybridoma cells. Biotechnol. Prog. 2000, 16, 847-853.

(17) Hjortso, M. A.; Nielsen, J . A conceptual model of autonomous oscillations in microbial cultures. Chem. Eng. Sci. 1994, 49, 1083-1095.

(18) J arzebski, A. B. Modeling of oscillatory behavior in continuous ethanol fermentation. Bi otechnol. Lett. 1992, 14, 137142.

(19) J ones, K. D.; Kompala, D. S. Cybernetic modeling of spontaneous oscillations in continuous culture of Saccharomyces cerevisiae. In AIChE Annual Mtg; Miami, FL, 1995.

(20) J ones, K. D.; Kompala, D. S. Cybernetic modeling of the growth dynamics of Saccharomyces cerevisiae in batch and continuous cultures. J. Biotechnol. 1999, 71, 105-131.
(21) Khibnik, A. I. Kuznetsov, Y. A.; Levitin, V. V.; Nikolaev, E. V. Interactive local bifurcation analyzer, 1992.

(22) Kuznetsov, Y. A. Elements of Applied Bifurcation Theory; Springer-Verlag: New York, 1995.

(23) Kuznetsov, Y. A.; Levitin, V. V. CONTENT: A Multiplatform Environment for Continuation and Bifurcation Analysis of Dynamical Systems; Centrum voor Wiskunde en Informatica: Amsterdam, Netherlands, 1997.

(24) Lenas, P.; Pavlou, S. Chaotic response of a periodically forced system of two competing microbial species. Chaotic Dynamics: Theory and Practice; Plenum Press: New York, 1992; pp 283-295.

(25) Li, J .; McLellan, P. J .; Daugulis, A. J . Inhibition effects of ethanol concentration history and ethanol concentration change rate on Zymomonas mobilis. Biotechnol. Lett. 1995, $17,321-326$.

(26) Lyberatos, G.; Kuszta, G.; Bailey, J. E. Bifurcation from the potential field analogue of some chemical reaction systems. Chem. Eng. Sci. 1985, 40, 1679-1687.

(27) McLellan, P. J .; Daugulis, A. J .; Li, J . The incidence of oscillatory behavior in the continuous fermentation of Zymomonas mobilis. Biotechnol. Prog. 1999, 15, 667-680.

(28) Munch, T.; Sonnleitner, B.; Fiechter, A. N ew insights into the synchronization mechanism with forced synchronous cultures of Saccharomyces cerevisiae. J . Biotechnol. 1992, 24, 299-313.

(29) Parulekar, S. J .; Semones, G. B.; Rolf, M. J .; Lievense, J . C.; Lim, H. C. Induction and elimination of oscillations in continuous cultures of Saccharomyces cerevisiae Biotechnol Bioeng. 1986, 28, 700-710.

(30) Pavlou, S.; Kevrekidis, I. G. Microbial predation in a periodically operated chemostat: A global study of the interaction between natural and externally imposed frequencies. Math. Biosci. 1992, 108, 1-55.

(31) Pavlou, S.; Kevrekidis, I. G.; Lyberatos, G. On the coexistence of competing microbial species in a chemostat under cycling. Biotechnol. Bioeng. 1990, 35, 224-232.

(32) Ramkrishna, D.; Kompala, D. S.; Tsao, G. T. Are microbes optimal strategists? Biotechnol. Prog. 1987, 3, 121-126.

(33) Srienc, F.; Dien, B. S. Kinetics of the cell cycle of Saccharomyces cerevisiae. Ann. N.Y. Acad. Sci. 1992, 59-71.

(34) Strassle, C.; Sonnleitner, B.; Fiechter, A. A predictive model for the spontaneous synchronization of Saccharomyces cerevisiae grown in continuous culture. II. Experimental verification. J . Biotechnol. 1989, 9, 191-208.

(35) von Meyenburg, H. K. Stable synchrony oscillations in continuous culture of Saccharomyces cer evi siaeunder glucose limitation. In Biological and Biochemical Oscillators; Chance, B., Pye, E. K., Shosh, A. K., Hess, B., Eds.; Academic Press: New York, 1973; pp 411-417.

(36) Zeike, H. R.; Zeike, C. L.; Ozand, P.T. Glutamine: A major energy source for cultured mammalian cells. Fed. Proc. 1984, 43, $121-125$.

(37) Zhang, Y.; Zhu, G.-Y.; Zamamiri, A. M.; Henson, M. A.; Hjortso, M. A. Cell population models for bifurcation analysis and nonlinear control of continuous yeast bioreactors. J . Process Control, in press.

(38) Zhou, W. Alteration of cellular metabolism by dynamic nutrient feeding. Cytotechnology 1997, 24, 99-108.

(39) Zhu, G.-Y.; Zamamiri, A. M.; Henson, M. A.; Hjortso, M. A. Model predictive control of continuous yeast bioreactors using cell population models. Chem. Eng. Sci. 2000, 55, 61556167.

Accepted for publication May 4, 2001.

BP010048W 